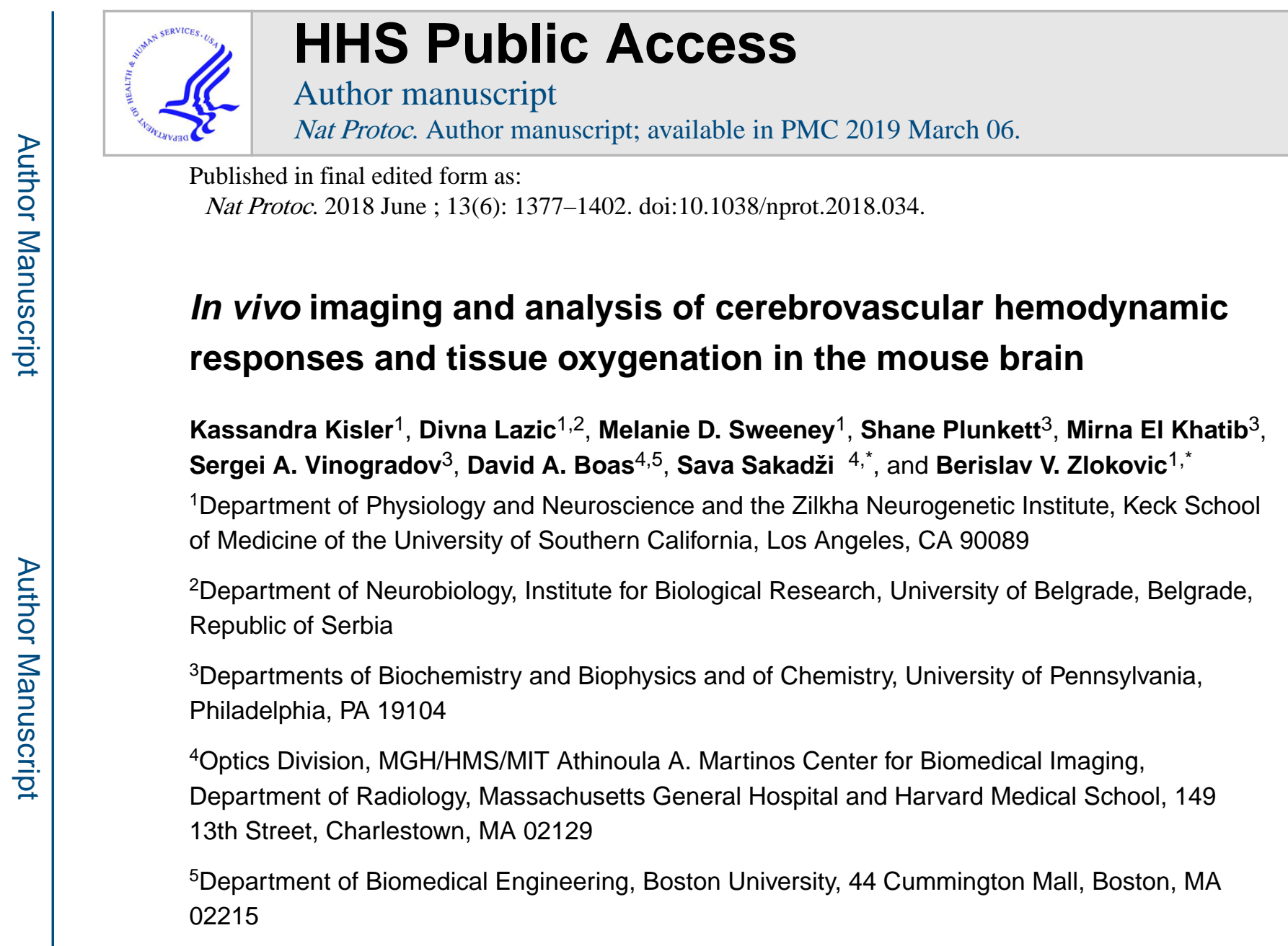

\begin{abstract}
Cerebrovascular dysfunction plays an important role in the pathogenesis of multiple brain disorders. Measuring hemodynamic responses in vivo can be challenging, particularly since techniques are often not described in sufficient detail and vary between laboratories. We present a set of standardized in vivo protocols that describe high-resolution two-photon microscopy and intrinsic optical signal imaging to evaluate capillary and arteriolar responses to a stimulus, regional hemodynamic responses, and oxygen delivery to the brain. The protocol also describes how to measure intrinsic nicotinamide adenine dinucleotide fluorescence to understand how blood $\mathrm{O}_{2}$ supply meets the metabolic demand of activated brain tissue and resting state brain tissue $\mathrm{O}_{2}$ concentration. These methods can detect cerebrovascular changes at far higher resolution than magnetic resonance imaging techniques, although the optical nature of these techniques limits their achievable imaging depths. Each individual procedure requires 1-2 hours to complete, with 2-3 procedures typically performed per animal at a time. These protocols are broadly applicable in
\end{abstract}

\footnotetext{
Address for correspondence: Berislav V. Zlokovic, M.D., Ph.D., Zilkha Neurogenetic Institute, 1501 San Pablo Street, Los Angeles, CA 90089, Phone: 323.442.2722 / Fax: 323.666.2184, zlokovic@usc.edu. AUTHOR CONTRIBUTIONS

K.K. and B.V.Z. conceived manuscript concept. K.K., S.S., and B.V.Z. contributed to experimental design. K.K. and S.S. performed experiments and analyzed data. D.L. and M.D.S. performed experiments and contributed to cranial window protocol development, S.P. and M.E.K. contributed PtP-C343 dye development. D.A.B. and S.A.V. contributed to project design. B.V.Z. contributed to project design and supervised project. K.K., S.S., and B.V.Z. wrote the manuscript with input from all authors. S.S. and B.V.Z. share senior authorship responsibilities.

*o-senior sharing authors

CONFLICT OF INTEREST

The authors declare that they have no competing financial interests.
} 
studies of cerebrovascular function in healthy and diseased brain in any of the existing mouse models of neurological and vascular disorders. All of these procedures can be accomplished by a competent graduate student or experienced technician, except the two-photon measurements of absolute $\mathrm{pO}_{2}$ level, which is better suited to a more experienced postdoctoral level researcher.

\section{Editorial Summary:}

A series of diverse in vivo imaging protocols for evaluating hemodynamic response and tissue oxygenation in mouse models of neurological and vascular disorders.

\section{Keywords}

mouse; brain; cerebrovascular hemodynamic responses; In vivo two-photon imaging; neurovascular coupling; capillary diameter; red blood cell velocity; intrinsic optical signal imaging; intrinsic nicotinamide adenine dinucleotide fluorescence; NADH; oxygen partial pressure; cranial window

\section{INTRODUCTION}

In humans, the brain makes up $2 \%$ of the body weight, but typically accounts for $20 \%$ of the body's net oxygen $\left(\mathrm{O}_{2}\right)$ and glucose consumption ${ }^{1-4}$. Accomplishing this task requires about $20 \%$ of cardiac output to maintain cerebral blood flow (CBF) through a vast network of arteries, arterioles, and capillaries in the brain ${ }^{3,5}$. CBF changes in response to local neuronal activity, increasing blood flow and dilating vessels in active brain regions to maintain sufficient $\mathrm{O}_{2}$ and nutrient supply during neuronal activity, a process called neurovascular coupling or functional hyperemia ${ }^{6}$. CBF dysregulation, vascular dysfunction and cerebrovascular disorder have been found to contribute to a growing list of human diseases and conditions including Alzheimer's disease (AD) ${ }^{6-10}$, amyotrophic lateral sclerosis $(\mathrm{ALS})^{11-13}$, frontal temporal dementia (FTD) ${ }^{14}$, Parkinson's disease (PD) ${ }^{15,16}$, Huntington's disease (HD) ${ }^{17,18}$, small vessel disease of the brain ${ }^{19,20}$ which contributes to more than 40 $50 \%$ of all dementias worldwide ${ }^{5,21-23}$, stroke $e^{5,23}$, hypertension $(\mathrm{HTN})^{24}$, diabetes $^{25}$, multiple sclerosis (MS) ${ }^{26}$, cardiovascular diseases ${ }^{27,28}$, traumatic brain injury ${ }^{29,30}$, psychiatric disorders ${ }^{31}$, and brain disorders associated with human immunodeficiency virus (HIV1) infection ${ }^{32}$.

Given the growing understanding of the importance of healthy vasculature in the maintenance of healthy brain function, there has been increasing focus on the measurement of hemodynamic and vascular responses in the brain in vivo in animal models to better understand the mechanisms behind healthy and diseased vascular function. Currently, rodent (typically mouse) models are most frequently used to study various aspects of these diseases in vivo. Interestingly, many of these models have been found to recapitulate various changes in vascular dysregulation identified in the human diseases, and vice-versa. For instance, in several AD mouse models, $\mathrm{CBF}$ changes were identified early in disease progression ${ }^{33-35}$. ALS, FTD, and HD mice exhibit regional changes in CBF, similar to observations in humans ${ }^{17,36,37}$. Angiotensin II-induced HTN and chronic HTN rodent models also exhibit reduced functional hyperemia ${ }^{24,38,39}$. 
Recent studies suggest that vascular dysregulation can contribute to onset and progression of multiple neurological disorders. However, the underlying mechanisms, contributing cell types, molecular pathways and potential therapeutic targets remain largely unexplored. As such, the need to accurately measure in vivo cerebrovascular responses in rodent models of neurological conditions is growing, and no longer relegated to a niche specialty.

Nevertheless, typical vascular imaging techniques used in living humans such as functional magnetic resonance imaging (fMRI) can be difficult and expensive to translate into mouse models. Methods have been developed over the years to measure different aspects of vascular and hemodynamic responses in rodent models. However, while working on our recent study ${ }^{40}$, and after discussion and feedback from our peers, we discovered that many of the techniques used to evaluate vascular effects have not been described in sufficient technical detail and with consistent protocols that could be easily reproduced by different labs. Thus, individual labs were left to develop methodological details and discover pitfalls by trial and error, resulting in wasted time and resources.

\section{Development and Overview of the protocols}

We have compiled a set of protocols for in vivo study of vascular measurements in rodent brain, recently used in our publication by Kisler et $\mathrm{al}^{40}$. In this study, we reported that pericytes, perivascular cells that enwrap brain capillaries, play an essential role in regulating capillary diameter and red blood cell (RBC) velocity changes in vivo in transgenic pericytedeficient mice. Moreover, using multiple additional techniques for blood flow measurements and oxygen delivery to the brain (that is blood flow dependent) we showed that a loss of pericytes leads to dysregulation of neurovascular coupling, reduced brain tissue oxygenation, and subsequent metabolic stress in the brain $^{40}$.

To assess changes in hemodynamic responses and vascular function in vivo in transgenic pericyte-deficient mice, we used an advanced methodology for high-resolution two-photon microscopy measurements of individual capillary and arteriole diameter responses ${ }^{40}$, and RBC velocity responses to a stimulus ${ }^{41}$ through a cranial window. We also used intrinsic optical signal (IOS) imaging for evaluation of regional hemodynamic responses and $\mathrm{O}_{2}$ delivery in response to stimulus ${ }^{42-45}$, and two-photon microscopy measurements of intrinsic nicotinamide adenine dinucleotide (NADH) fluorescence in response to stimulus, which indicates how well blood $\mathrm{O}_{2}$ supply meets the increased metabolic demands of the stimulated brain tissue ${ }^{46,47}$. We also used two-photon imaging to evaluate local resting state tissue $\mathrm{O}_{2}$ partial pressure $\left(\mathrm{pO}_{2}\right)$ using a phosphorescent dye $\mathrm{e}^{48}$. These methods are shown schematically in Figure 1.

While some of these techniques require specialized equipment, many of these measurements are straight forward and the necessary equipment can be readily obtained. Our goal here is to provide a set of protocols (Figure 1) that might bring some unity to the study of cerebrovascular dynamics by providing standard protocols and theoretical background to work from, and at the same time provide a solid guide for many laboratories interested in incorporating vascular dynamics measurements into their ongoing or future studies. In the experimental design, we provide discussions of the background and theoretical considerations that the experimenter should be mindful of when planning these experiments, 
as well as practical tips we have learned doing the experiments, and a discussion of possible alternative approaches to each respective technique.

\section{Applications of the protocols}

The techniques we describe here, used individually or in combination, have the potential for broad application to examine vascular physiology and pathophysiology, and effects on brain tissue metabolism and oxygenation in multiple rodent models of neurological and systemic disorders affecting brain functions. While our present examples focus on models of pericyte dysfunction ${ }^{40}$, scientists who study any of the neurovascular unit-related cell types could find these techniques useful. For example, these protocols can be applied to models of AD that have all been shown to develop a prominent cerebrovascular dysfunction, including models carrying different human mutations in the amyloid precursor protein $(A P P)$ gene and presenilin (PSEN1) gene causing autosomal dominant $\mathrm{AD}^{49}$, mutations in Tau gene causing microhemorages, BBB dysfunction and tau accumulation that model AD and FTD pathophysiology 50,51 , apolipoprotein E humanized transgenic models carrying major genetic risk factor for sporadic late-onset $\mathrm{AD}^{52,53}$, ALS models, as well as models of $\mathrm{PD}, \mathrm{HD}$, and other neurodegenerative disorders, as summarized recently $6,10,54$. These techniques could also be employed to investigate acute vascular changes developing in rodent models of ischemic stroke and microinfarcts ${ }^{55,56}$, hemorrhagic stroke ${ }^{57}$, traumatic brain injury ${ }^{58}$, and vascular risk factors such as, to name a few, hyperhomocysteinemia ${ }^{59,60}$, diabetes ${ }^{61}$, cardiac $\operatorname{arrest}^{62}$ or aberrant angiogenesis ${ }^{63}$. These techniques will also be applicable to transgenic models of rare, monogenic human neurological disorders with primary genetic effects in non-neuronal cells of the neurovascular unit ${ }^{64}$ such as haploinsufficiency in the Slc2a1 gene encoding glucose transporter 1 (GLUT1) leading to brain endothelial-specific reductions in GLUT1 ${ }^{65}$, mutations in major facilitator superfamily domain-containing protein 2a (MSFD2A) gene causing microcephaly, cognitive and motor dysfunction, and BBB disruption ${ }^{66,67}$, mutations in the Notch3 gene leading to specific degeneration of brain vascular smooth muscle cells and pericytes resulting in the most common genetic cause of stroke known as cerebral autosomal dominant arteriopathy with subcortical infarcts and leukoencephalopathy (CADASIL) ${ }^{68,69}$. They are also applicable to brain tumor models ${ }^{70}$, and/or any models of systemic diseases with effects on brain function.

With a sealed cranial window, most of these measurements could also be performed longitudinally, opening up the possibility to study a disease or treatment progression over time. These techniques are also not limited to imaging the brain, and could be adapted to imaging studies in cochlea ${ }^{71}$ and spinal cord ${ }^{72,73}$ as well, with the appropriate preparations.

We anticipate that this set of protocols will be a valuable tool for those studying vascular function in the healthy and diseased rodent brain, including any of the existing mouse models of neurological and/or vascular disorders, as well as the potential to evaluate the vascular effects of new therapeutics in vivo. Furthermore, while we have presented techniques here for use with anesthetized mice, many of these protocols could be adaptable for studies in awake animals with appropriate head stabilization and choice of stimulus, which will avoid a use of anesthetics as a potential confound. 


\section{Limitations}

Because the imaging techniques we describe here are all optical methodologies, imaging depths through the brain are limited mainly to the upper layers of the cortex and deeper brain regions are not accessible. Brain tissue absorbs and scatters the light passing through it, reducing the number of photons detected as the imaging depth increases. Thus, imaging depth is essentially limited by the signal-to-noise ratio (SNR), although new detectors and fluorescent markers are continuously being developed in an attempt to overcome this issue. Magnetic resonance imaging (MRI) is capable of imaging through the whole brain and report regional $\mathrm{BBB}$ integrity and $\mathrm{CBF}$ perfusion information, overcoming the imaging depth $^{74}$. However, MRI image resolution is severely limited compared to optical imaging methods. Of the techniques presented here, two-photon techniques afford the greatest depth resolution. For diameter and velocity measurements, we have consistently performed imaging to about $400-500 \mu \mathrm{m}$ depth (roughly mouse cortex layer IV) through a cranial window, although others have reported high resolution functional vascular imaging from greater depths ${ }^{75}$, while for NADH imaging and absolute $\mathrm{pO}_{2}$ imaging using $\mathrm{O}_{2}$-sensitive dye PtP-C $343^{48}$, depths are typically limited to the upper $200-300 \mu \mathrm{m}$ of cortical tissue ${ }^{76}$. It is possible to image a little deeper, to about $450 \mu \mathrm{m}^{77}$, if intravascular $\mathrm{pO}_{2}$ is measured. These limitations are mainly due to increased contribution of the out-of-focus signal generation at greater depths, so it is best to stay in the regions where this contribution is minimal. NADH and $\mathrm{pO}_{2}$ imaging also tend to have lower temporal resolution than the other techniques we describe here, which can limit their use in observing fast dynamic changes in tissue or vessels. IOS imaging essentially measures surface and near-surface tissue changes, although it is estimated that a small fraction of the IOS signal is produced from deeper scattering and reflectivity ${ }^{43}$. Supplemental table 1 summarizes imaging depths, resolution, and acquisition rate parameters for each imaging method described in this protocol.

\section{EXPERIMENTAL DESIGN}

\section{Local capillary diameter and RBC velocity measurements by two-photon microscopy (step 18B)}

Theoretical considerations-In our recent paper $^{40}$, we detected capillary diameter changes as small as $1 \%$ relative to the baseline diameter with our two-photon microscope in the somatosensory cortex of anesthetized mice. Capillary diameter changes that are much smaller than the optical resolution of a standard two-photon microscope can be measured effectively by oversampling and averaging the linescans. Signal averaging increases the SNR and can bring the desired signal out of the noise. This is combined with oversampling the image or line scan, which in this case means using pixels of minimal size, maximizing the number of pixels making up the image or linescan, to help resolve the signal. These concepts were formalized mathematically by Shahram and Milanfar ${ }^{78}$ and similar conclusions drawn later by Ram, et al $^{79}$, where they identify SNR and pixel resolution as key elements for resolving distances below the limit described by Rayleigh ${ }^{80}$ (see also https:// www.microscopyu.com/techniques/super-resolution/the-diffraction-barrier-in-opticalmicroscopy). 
Intertwined with the spatial resolution is temporal resolution. To resolve temporal diameter changes, it is important that the linescan rate exceeds the rate of diameter changes. To avoid aliasing, the minimum linescan rate should be more than twice the rate of the fastest change one is trying to resolve $\mathrm{e}^{81-83}$. There is sufficient in vivo data on capillary diameters to indicate that capillary diameter changes occur on the order of $1 \mathrm{~s}^{40,84-90}$, so the minimum linescan rate should be greater than $2 \mathrm{~Hz}$. However, acquiring data at much higher rates both ensures sufficient time resolution to accurately reconstruct the capillary diameter time course, and allows us to take advantage of averaging to increase SNR.

Brain capillary diameter measurements-The diameter data was acquired using 1D line scans (lines of pixels) of fluorescein isothiocyanate (FITC)-dextran (70 kDa)-labeled blood plasma in capillaries (Fig. 2a) and arterioles, not 2D images as was done in several other studies $84,85,91,92$. Because capillaries are small, $~ 3-6 \mu$ m diameter, it can be technically challenging to achieve both sufficient temporal and pixel resolution to resolve capillary diameter changes using 2D imaging. By employing line scans, we could take better advantage of the key concepts we outlined above (high resolution, SNR, and speed) within the performance constraints imposed by our microscope system. For diameter measurements, line scans were taken perpendicular to the vessels at $15 \mathrm{~Hz}$, which is similar to other reported studies using line scans ${ }^{75,87,89,93}$, but faster than in most other recent studies relying mainly on $2 \mathrm{D}$ images ${ }^{84,85,91,92}$. Line scans allowed us to oversample the vessel diameter with a good temporal resolution and high pixel resolution, typically on the order of 20-50 nm/pixel depending on the microscope and size of the vessel measured. This gave us enough pixels to detect small changes in diameter after the data has been processed as detailed below.

In our experiments, we recorded capillary diameter line scans in response to a $10 \mathrm{~s}$ long electrical stimulus applied to the hind limb. For ease of analysis, all the line scans recorded from an individual capillary diameter experiment time course are built up into an image, or "kymograph," where the vessel diameter was recorded as a line of pixels in one axis, and time in the other (Figure 2a,b). The data then undergoes 2D Gaussian filtering to reduce noise from contrast fluctuations due to RBC passage through the vessel and background outside the vessel ${ }^{83}$, and thresholding ${ }^{94}$ to differentiate the vessel signal from the background to yield a black and white image of the capillary diameter vs. time (see Protocols section steps 18B.xiii-xiv for details). The Gaussian filter is relatively mild, only a 3-point filter (see Protocols section step 18B.xiii), and acts on both the time component (horizontal axis in the figure $2 \mathrm{~b}$ example) and spatial component of the kymograph (vertical axis in figure $2 \mathrm{~b}$ example), yielding a cleaner image upon thresholding. The diameter information is then extracted from every line in the thresholded image to build up the "Raw" diameter time course trace (Figure 2c). To reduce higher frequency noise and breathing artifacts, additional 1D low pass, notch (a type of filter to remove very select frequency ranges -- used to remove the breathing artifact frequency here), and 10-15 point ( 1 s window) box filters are applied to the timecourse trace, resulting in a final "Filtered" diameter trace vs time (see below for details). Figure $2 \mathrm{~d}$ illustrates the final filtered diameter trace from a capillary with $\sim 3 \%$ peak dilation in response to stimulus. 
Often the desired diameter measure outcome is a time to some percentage of peak dilation. However, even with the filtering, there is still some noise remaining in the diameter trace, which means there can be some ambiguity regarding the peak dilation level. To compensate for this, we chose to fit sigmoid curves to the individual diameter dilation time course traces, and used the fits to extract the times to percentage peak. In our case, we chose to look at time to reach $50 \%$ peak dilation (Figure 2e). While the dilation time course could likely be described by more complex functions, the sigmoid shape recapitulated the characteristics of the diameter increase and tolerated a moderate amount of noise in the data, and is consistent with other simplified fitting schemes used previously ${ }^{85,89}$. This technique helped minimize any uncertainty in the $50 \%$ peak time measurement that could come from the remaining noise in the dilation time course. This process is repeated for all capillary measurements, and then averaged per mouse.

In Figure $2 \mathrm{f}$ we illustrate this process for the capillary diameter dilation time course measurements for one mouse, where the mouse average capillary diameter sigmoid response curve was derived from the individual capillary sigmoid responses. The data was quantified by averaging the time to $50 \%$ peak dilation from sigmoid fits to individual capillaries to obtain the 50\% peak dilation average per mouse, then mouse average values were compared across experimental conditions.

The advantage of this particular technique using high resolution rapid line scans, filtering, and fitting, is the ability to resolve small diameter changes in small vessels (capillaries) less than $5 \%$. The same process can be used to measure diameter changes in response to stimulus in arterioles, as we reported ${ }^{40}$. Because these vessels are larger, it is often easier to measure diameter changes. In our study, for arteriole diameter measurements we used the same pixel sizes and acquisition speeds as for the capillary measurements and performed the same analysis steps.

Red blood cell velocity measurements-For RBC velocity measurements, high speed two-photon line scans along the axis of the vessel (Figure 3a) are acquired to image RBC movement through capillaries in response to a stimulus as for the diameter measurements (10 s electrical hind limb stimulus). Here, scan speed and SNR are more critical then resolution, which makes this measurement less challenging then capillary diameter measurements. Because of the scan rates required (typically $>650 \mathrm{~Hz}$ ) RBC velocity measurements are almost always performed as line scans (e.g. ${ }^{40,75,84,87-89,93}$ ). The idea is to catch the same RBC in multiple lines of the scan to build up a time course of the RBC passage through the vessel segment imaged. As with the diameter measurements, the line scans are built up into kymographs. Here, the RBCs appear as shadows (dark spots) in the dye-labeled blood plasma, and form stripes in the kymograph that track their movement through the vessel (Figure 3b). The angle of the RBC stripes is an indicator of the RBC speed in the vessel, but present analysis methods rely on sophisticated algorithms that provide better accuracy then measuring RBC stripes by hand (e.g. ${ }^{41,95}$ ). For our data, a Matlab program published by Kim, et $\mathrm{al}^{41}$ provided a straight forward means to extract the raw velocity time course from the images (Figure 3c). Briefly, Kim's program runs a crosscorrelation routine between lines in the kymograph to determine the $\mathrm{RBC}$ velocities. The program tolerates a wide range of $\mathrm{RBC}$ velocities and SNR, and has adjustable settings to 
customize the analysis to the imaging speed used and vessel type (capillary or arteriole) imaged. It's worth noting that the raw data traces produced by the Matlab routine typically exhibit a significant heartbeat artifact (Figure $3 \mathrm{c}$ inset; see also ${ }^{41,93,96}$ for examples). While this means that subsequent filtering is required to remove the artifact, it also indicates that the data acquisition was good (there should be a heartbeat artifact) and Matlab analysis parameters were set appropriately. The resulting raw RBC velocity data is normalized to its baseline value, filtered with low pass, notch, and box filters (Figure 3d), and fitted with sigmoid curves similar to the capillary diameter measurements (Figure 3e). Figure $3 \mathrm{f}$ illustrates a set of capillary RBC velocity measurements from one mouse. Similar to capillary diameter sigmoid curves, the average RBC velocity increase time course for this mouse was derived from the individual RBC velocity sigmoid curves. We calculated the time to $50 \%$ peak RBC velocity for each individual capillary velocity sigmoid curve, and averaged the values per mouse to compare across experimental conditions. The same procedures are used to measure arteriole RBC velocity as well. However, in our hands this analysis works better on small to medium arterioles (up to $\sim 25 \mu \mathrm{m}$ diameter) within the brain parenchyma where fewer RBCs pass through the vessel cross section at the same time. Although it is possible to measure RBC velocity from larger vessels ${ }^{41}$, vessels with larger diameters (>25 $\mu \mathrm{m}$ ) typically have many RBCs passing through the vessel at once, which could potentially lead to inaccurate results.

Additional technical considerations-Noise sources and the need for filtering: There are many sources of noise that can find their way into the image information acquired. Some of these sources are readily identifiable such as heart beat and breathing artifacts because a live animal is being imaged (see the data trace period between about $1.5-5 \mathrm{sec}$ in figure $2 \mathrm{c}$ for example). The breathing artifact should be apparent when looking at the frequency spectrum of the data trace as well. In addition to these noise sources, many other noise sources can contribute to the overall signal, including small mechanical vibrations or movements which could be due to equipment on the microscope table, inadequate fixation of the mouse head, incomplete vibration isolation, room air flow, etc (for a nice discussion see e.g. https://www.photonics.com/EDU/Handbook.aspx?AID=25517), electrical noise from the equipment and detectors themselves, and optical noise from varying numbers of photons detected, or light in the imaging room (including monitor screens), and thermal variations ${ }^{97}$. One tries to minimize these disturbances as much as possible, but even in an ideal situation there will be some noise.

In our hands, the variance over the normalized baseline diameter measurement was on the order of $10^{-4}$ to $10^{-5}$. Visually this translates to normal oscillations due to breathing artifacts of up to $\sim 1 \mu \mathrm{m}$ in the plane of the linescan. The vessel analysis tolerates this motion because a shift of the vessel in the plane does not alter the relative positions of the edges of the vessel. Shifts out of the imaging plane are more problematic. This typically manifests as oscillations in diameter measured. If the oscillations are small, the image processing we describe can minimize the effect. For data with strong oscillations, the best course is to discard it.

To help minimize artifacts, one of the most important aspects is to ensure that the mouse's head is securely fixed in position, and that the platform(s) on which the mouse and 
microscope sit are very stable. Here, imaging fixed tissue slides at high resolution and magnification may help identify vibration issues with the equipment itself. The Kopf frames (see Equipment section) have turned out to be surprisingly stable for head fixation compared to other fixation methods we tried. Keeping a constant level of anesthesia is also important. Variations can result in subtle changes in vessel size and position, which can result in focal drift during diameter and velocity measurements. When the animal is moved from one location to another (for example from IOS to two-photon microscopes), or a refill of the anesthetic reservoir is needed, we found it is best to allow the animal to stabilize for several minutes before imaging.

Minimizing noise sources and maximizing SNR help when acquiring data, but there are limited options to deal with noise recorded with the signal afterwards. Filtering is often the option we have the best control over ${ }^{83}$, and is commonly used to help distinguish the signal of interest from noise and background (for examples see $84,85,87,89,93$ ). However, the filtering method and parameters used (strength, threshold level, number of points or time window, etc) should be considered carefully in order to avoid filtering away the signal of interest.

While we presently lack sufficient experience with these measurements in awake animals to say with certainty that identical filtering routines described here, including the notch filter, can be applied to awake, freely breathing animals, inspection of the data, and the data frequency spectrum should help inform as to whether a notch filter would be appropriate, and which frequency to apply it at.

Igor Pro, a programmable analysis software, was used to automate several of the image processing and analysis steps described in these diameter and RBC velocity protocols, but the same processes could be implemented in other software platforms such as Matlab.

Often, we measure diameter and RBC velocity in the same vessel segment, just changing the orientation of the line scan and acquisition parameters for the appropriate measurement. For both measurements, it is important to consider the orientation of the vessel. Vessels oriented parallel to the image plane are easiest to measure, but it is important to focus the image at the vessel center where the width of the vessel is greatest to ensure accurate measurements. Diameters of larger vessels (arterioles) that are oriented perpendicular to the image plane are also possible to measure, but RBC velocity cannot be measured in that orientation. Vessels oriented at an angle to the image plane present difficulties as there could be movement on more than one axis. Even when taking all these factors into consideration, typically only about $50-60 \%$ of the data recorded can be used because of the noise and artifacts present in some signals.

We also want to emphasize the importance of keeping track of location in the vascular tree, and which vessels are being imaged. We have found that sketching the vasculature studied, marking measurement locations in sketches and/or screen shots (Microsoft Paint works well for this), and taking quick z-stacks help in tracking the details during the experiment.

To coordinate stimulus with image acquisition, we used a digital-to-analog converter (DAC) and accompanying software that was programmed to output a trigger to an electrical stimulus source attached to the animal. Simultaneously, the DAC records the trigger signal 
from the microscope, indicating the start of imaging or linescan acquisition. Later, this information was used to find the exact timing of the stimulus relative to the image acquisition. In order to determine the brain region activated by the stimulus, IOS imaging is performed prior to two-photon imaging.

Comparison to Alternative Methods-As we described, it can be challenging to achieve both sufficient spatial and time resolution with 2D scans. Linescan and 2D imaging should be achievable with a confocal system as well, but imaging depth would be essentially limited to surface vasculature. Another technique is optical coherence tomography (OCT), which has the capability to rapidly image large areas of brain tissue at greater depths than two-photon imaging ${ }^{98}$. This has the potential to be a highly useful technique, but at present it is unclear whether OCT can achieve sufficient spatial resolution to image small changes in capillary diameters, while maintaining the advantage of the rapid volumetric scans.

Acquisition of RBC velocity data from individual vessels using two-photon microscopy tends to be more consistent across labs. The differences between techniques arise mainly from the choice of analysis method. The two most common analysis methods rely on either cross-correlation $^{41}$, which we have chosen to use, or radon transform algorithms ${ }^{95}$. For our purpose, the cross-correlation analysis was easier to implement with our data. OCT may also be capable of measuring capillary RBC velocity changes ${ }^{98,99}$.

\section{Intrinsic optical signal (IOS) imaging in green and red (step 18A)}

Theoretical considerations-Intrinsic optical signal (IOS) imaging uses diffusely reflected light to detect spatially resolved regional changes in oxygen saturation of hemoglobin (the blood oxygen carrier) or total hemoglobin content in response to brain activation. Light scattered back from the surface and upper layers of the brain is imaged over time, and subsequent image analysis reveals the location, amplitude, and time course of changes in the tissue optical properties, the "IOS signal" 42 . In vivo, the IOS signal in response to functional brain activation is mainly a result of changes in the tissue optical absorption due to the changes in the signals originating from oxyhemoglobin and deoxyhemoglobin. Changes in oxy-and deoxyhemoglobin can arise from variations in blood flow, blood volume, and cerebral metabolic rate of $\mathrm{O}_{2}$ consumption. Oxy- and deoxyhemoglobin have different optical absorption spectra (Figure 4a) and thus provide intrinsic optical contrast that can be used to measure different aspects of hemodynamic response to stimulus ${ }^{43}$. At $\sim 530 \mathrm{~nm}$ (green light), the molar extinction coefficients of oxyand deoxyhemoglobin are nearly identical (Figure 4a). Thus, IOS changes at this wavelength reflect changes in the total hemoglobin content in the brain tissue, implying changes in the blood flow and blood volume (due presumably to vessel dilation) in response to stimuli $^{43,44,100}$. At $530 \mathrm{~nm}$, the IOS signal is mostly monophasic with a negative deflection, since optical absorption transiently increases during stimulus. Figure $4 \mathrm{~b}-\mathrm{c}$ shows an example of a green IOS experiment and signal trace from a wild type mouse.

At $\sim 630 \mathrm{~nm}$ (red light), the extinction coefficients of oxy- and deoxyhemoglobin are different (Figure 4a). The diffusely reflected red light is mostly due to oxyhemoglobin ${ }^{43-45}$, while the absorption is due to deoxyhemoglobin. Functional hyperemia leads to a transient 
decrease in deoxyhemoglobin concentration, weakening absorption, and consequently the active areas get transiently brighter, generating a positive peak, or "overshoot," signal. Our experiments focus on this positive peak, which indicates deoxyhemoglobin washout (Figure 5), as a measure of the balance between oxyhemoglobin supply vs. demand in response to stimulus.

Finally, it is worth noting that the amplitudes reported for red and green IOS signals in mice vary substantially across the literature, sometimes making it difficult to compare different data sets. Some of this is clearly attributable to the many variations in experimental designs and stimulus paradigms reported, but we have also noticed that in our hands, the strain of the mouse used in the study plays a role in the size of the response observed as well (K.K. unpublished observation).

IOS imaging - In our setup, light from an LED array is reflected from the brain surface through a dissecting scope fitted with a bandpass filter to a high-speed camera, where the image of the brain is recorded over time (Figure 6). Our set up was inspired by descriptions provided by Harrison, et $\mathrm{al}^{42}$, but with an eye toward simpler implementation. We use a $2 \times 3$ linear array of LEDs mounted on a post and angled toward the mouse's head as our light source. When positioned correctly, there is no issue with shadows in the cranial window area, and the window is evenly lit. Most dissecting scopes with camera ports do not have dedicated filter holders, but do have a space between the tube lens and camera mount large enough to position a 15-18 $\mathrm{mm}$ diameter emission filter (Figure 6). We chose a camera with good sensitivity, dynamic range, and speed that could also be used for other applications such as voltage sensitive dye imaging (for examples see $\mathrm{e}^{40,101}$ ). In our studies we used a 12bit camera with 12,000 to 100,000 electrons well depth depending on settings used, and dynamic range of $\sim 60 \mathrm{~dB}$. We ran this camera at $33 \mathrm{fps}$, but anything over $10 \mathrm{fps}$ should have sufficient time resolution to capture the IOS signal time course ${ }^{42}$. Conveniently, this camera was packaged with software designed to analyze IOS signals (see Materials section). Some cameras may require additional software or programming to analyze IOS data as described here and in the protocol (steps 18A.x-xiii), depending on software features packaged with the camera. This allows almost instant preliminary processing and display of the IOS signals, which helps to guide subsequent imaging.

In practice, for either red or green IOS, one data set is made up of an average of multiple trials in response to stimulus to achieve a robust signal. We typically use 10 trials, but reports in the literature have ranged from 6 to 100 trials per data set to obtain good SNR, with 10-20 trials most typical. For analysis, an ROI location is chosen such that it is not near any large surface vessels, but as close as possible to the maximum signal location (Figure 4a, 5a). This helps to ensure that the signal obtained is generated by the vascular response in the brain parenchyma. IOS signals are often expressed as percent change in reflected intensity $(\Delta \mathrm{I})$ as a fraction of baseline intensity $\left(\mathrm{I}_{\mathrm{O}}\right)$, or $\Delta \mathrm{I} / \mathrm{I}_{\mathrm{O}}, \%$. From the time courses determined from the ROIs (Figure 4c, 5b), information such as time to peak signal (or a percentage of peak signal) and peak amplitude can be determined and compared against different experimental conditions. 
While there is a range of stimulus durations reported for IOS studies, we often try to use short stimulus durations, $<1 \mathrm{~s}$. Experiments requiring lengthy stimulus trains as used in our recent paper to match our capillary diameter and velocity imaging stimulus (Figure 4, $10 \mathrm{~s}$ stimulus; ${ }^{40}$ ) require longer recording times. In the case of a $10 \mathrm{~s}$ stimulus, a single data set took about 15-20 min to complete. In this case, each cycle had a $40 \mathrm{~s}$ duration recording and an additional $70 \mathrm{~s}$ delay between recordings.

For two-photon diameter, RBC velocity, and NADH measurements, IOS data is acquired first, to determine the brain location responsive to the stimulus. For this purpose, we typically make a sketch or take a screen shot of the surface vasculature, with the active area marked. This information then accompanies the mouse to the next experiment and acts as a map for finding the correct imaging location.

Comparison to Alternative Methods-IOS imaging can be implemented at single wavelengths as we describe here, or with additional software controls, combined for simultaneous imaging at multiple wavelengths ${ }^{42}$. Techniques for measuring relative CBF (rCBF) such as Laser Speckle Flowmetry (LSF) and Laser Doppler Flowmetry (LDF) can be used to assess rCBF during whisker or forepaw stimulation. They provide a complementary information to the IOS imaging at $530 \mathrm{~nm}$, since CBV and CBF changes are typically strongly positively correlated during functional hyperemia. LDF is likely the simplest of these three methods to set up, but it does not yield images of the measurement region like IOS and LSF imaging.

\section{Two-photon NADH measurements (step 18C)}

Theoretical considerations-NADH is present in all tissues in the body, and is involved with metabolic processes within cells. Both NADH and its close relative, nicotinamide adenine dinucleotide phosphate (NADPH) are intrinsically fluorescent in their reduced states, but not their oxidized states $\left(\mathrm{NAD}^{+}\right.$and $\left.\mathrm{NADP}^{+}\right)$. For practical measurement purposes, we consider only NADH, which is present at much higher concentrations then NADPH in tissue ${ }^{47,102}$. NADH fluorescence intensity exhibits profound increase as tissue $\mathrm{O}_{2}$ concentration abnormally decreases below $3-5 \mathrm{mmHg}^{76}$, and as such NADH fluorescence can be used as a marker of metabolic changes in vivo ${ }^{46,47,103,104}$. The exact dependence of NADH fluorescence intensity on tissue $\mathrm{O}_{2}$ concentration within the normal range of tissue $\mathrm{pO}_{2}$ and different brain activation states is not yet fully understood. Nevertheless, it is generally accepted that it exhibits negative correlation with the tissue $\mathrm{pO}_{2}$ in situations with abnormal tissue oxygenation. Because NADH fluoresces in the blue region of the spectrum, its fluorescence is easily absorbed by hemoglobin, which can affect measurements of NADH fluorescence in the presence of blood flow changes. To compensate for this effect, we use a "non-functional" fluorescent marker, SR101, in the brain to correct for hemodynamic effects on the NADH signal, as previously reported ${ }^{105}$. Studies using numerical simulations that were then tested and confirmed with experimental data ${ }^{105}$ arrived at a scaling factor equal to 1.15 (see step 18C.xv for use in analysis) to correct for hemodynamic effects in two-photon NADH images using the SR101 signal. 
NADH imaging - In this protocol we use two-photon microscopy to image changes in NADH fluorescence in response to a hind limb stimulus as used for capillary diameter measurements, which gives a measure of how well blood oxygen supply meets the increased metabolic demands of the stimulated tissue $76,90,105$. The NADH signal is somewhat weak compared to extrinsic dyes, and appears as diffuse fluorescence throughout the image with shadows where the blood vessels are located (Figure 7a).

We also image SR101 labeled astrocytes and oligodendrocytes (Figure 7a). Conveniently, SR101 can be topically applied directly to the brain and its fluorescence can be excited with the same two-photon wavelength as for NADH imaging. NADH images are acquired over time in response to a stimulus, and these images are interleaved with images collected of SR101 fluorescence. Preferably, if the microscope can be configured appropriately the NADH and SR101 fluorescence can be acquired simultaneously. We extract the image intensity data in ImageJ using regions of interest (ROIs) drawn to avoid the vessel shadows, and normalize each fluorescence channel trace to its pre-stimulus basal value. Following a simple correction method using a scaling factor as described ${ }^{105}$, we used the SR101 fluorescence trace to correct the NADH fluorescence to arrive at the final NADH signal time course in response to stimulus. Briefly, the method involves multiplying the normalized SR101 signal by a scaling factor and then subtracting the result from the normalized NADH signal. Figure $7 \mathrm{a}$ and $\mathrm{b}$ illustrate the corrected NADH response to a $10 \mathrm{~s}$ long hind limb stimulus in a platelet-derived growth factor receptor beta (Pdgfr $\beta+/-)$ deficient mouse, which we have found to exhibit a mismatch between metabolic supply and demand due to changes in neurovascular coupling ${ }^{40}$. For comparison, we also plot the time course of NADH fluorescence from a control littermate animal, which exhibits a much smaller NADH fluorescence change (Figure 7b). Some have reported seeing Krogh cylinders, sharply lower NADH fluorescence regions around arterioles in the mouse cortex, where tissue oxygenation is likely higher ${ }^{76}$, but others, in rats, do not show such prominent features (e.g. ${ }^{105}$ ). Working with the interleaved images provided a bit of a challenge, as the NADH and SR101 channels essentially had different time courses. To compensate for this, we interpolated the points in the SR101 trace to match the NADH time points before performing the correction. While many programs are capable of this, we found that Igor Pro has built in routines to interpolate data automatically as it performs such calculations.

Prior to two-photon imaging, IOS imaging is performed in order to determine the brain region activated by the stimulus. We use identical stimulus parameters as for the diameter and velocity measurements. With a little practice, the NADH measurements go quickly, and the animal could be used for other measurements.

Comparison to Alternative Methods-Intrinsic NADH fluorescence imaging using two-photon microscopy allows for image acquisition at sub-surface depths, using longer excitation wavelengths compared to other methods. Other intrinsic NADH imaging approaches utilize single-photon excitation at blue and near-UV wavelengths to excite NADH fluorescence ( $\mathrm{se}^{47}$ for a historical review of these techniques). These single-photon techniques are easy to implement but provide very limited imaging depth in vivo. The excitation wavelengths used are also more likely to cause damage to the tissue. More recently, genetically encoded sensors have been developed for in vivo NADH imaging (see 
e.g. ${ }^{106,107}$ ). This technique is promising, but requires a means of expressing the sensor in the model of interest.

\section{Two-photon absolute oxygen partial pressure $\left(\mathrm{pO}_{2}\right)$ measurements.}

Theoretical considerations and background-Quantitative measurements of oxygen concentrations in biological environments can be accomplished by measuring phosphorescence lifetimes of exogenous phosphorescent probes ${ }^{108}$. Similar to fluorescence lifetime imaging (FLIM), where the fluorescence lifetime yields information about the fluorophore microenvironment ${ }^{109}$, phosphorescent lifetimes of $\mathrm{O}_{2}$-sensitive probes yield information about the local absolute $\mathrm{O}_{2}$ concentrations. Unlike fluorescence, where light emission occurs on the order of nanoseconds after excitation, phosphorescence light emission typically occurs on the order of microseconds. The phosphorescence lifetime of an $\mathrm{O}_{2}$ probe is inversely proportional to the partial pressure of $\mathrm{O}_{2}\left(\mathrm{pO}_{2}\right)$ in the immediate vicinity of the probe molecule, providing means for spatially localized measurements of dissolved $\mathrm{O}_{2}{ }^{48,108}$

In the past, this technique has been used to image both cerebral intravascular and tissue oxygenation with wide field with charge-couple device (CCD) cameras ${ }^{110-112}$ or, alternatively, by means of confocal microscopy ${ }^{113}$. Combining the phosphorescence quenching approach with two-photon microscopy ${ }^{48}$ makes it possible to image cortical $\mathrm{O}_{2}$ delivery and consumption in tissue and microvasculature in depth-resolved fashion while retaining micron-scale spatial resolution ${ }^{114,115}$. The later technique has been termed TwoPhoton Phosphorescence Lifetime Microscopy (2PLM) of oxygen, and it has become a valuable tool in neuroscience ${ }^{40,77,116-118}$ and stem cell biology ${ }^{119}$.

Phosphorescent probes for 2PLM, e.g. PtP-C $343^{48}$, have been constructed specifically with two-photon excitation regime in mind and optimized for stable, calibration-free measurements in complex biological environments. At the core of the $\mathrm{O}_{2}$-sensitive phosphorescent probes is a highly phosphorescent Pd or Pt porphyrin. Upon excitation, porphyrin undergoes fast intersystem crossing into its triplet state and emits phosphorescence, which is quenched by molecular oxygen in a diffusion-controlled manner which can be mathematically described using a Stern-Volmer relationship. The PtP-C343 probe used in this protocol belongs to the family of dendritically protected phosphorescent probes. The porphyrin at its core (PtP) is encapsulated inside poly(arylglycine) dendrimer with coumarin-343 "antennae" to enhance absorption of two-photon wavelengths. The molecule is further encapsulated in poly(ethylene glycol) (PEG), preventing interactions with endogenous components of the biological system and making the probe's signal stable and selective to oxygen. Unlike measurements based on the difference between the optical absorption of oxy- and deoxyhemoglobin, 2PLM, as well as other phosphorescence-lifetimebased techniques, is insensitive to changes in the optical properties of the tissue. Importantly, oxygen consumption by the 2PLM is extremely small and so far we have not seen its effect on our measurements. Singlet oxygen produced during the quenching of the probe's triplet state rapidly (within $\sim 3 \mu$ s) relaxes back to the ground state. During its lifetime, endogenous biological substrates can react with singlet oxygen, potentially leading to its consumption. The rate of oxygen diffusion in aqueous environments is high, though, resulting in 
replenishment of oxygen in the excitation volume between excitation pulses. Also, because the two-photon excitation effectively results in a highly confined excitation volume, the risk of photodamage is greatly reduced. As a result, singlet oxygen generation is minimal with this technique, and previous tests of cell viability after tissue $\mathrm{pO}_{2}$ measurement showed no evidence of phototoxicity ${ }^{114}$. The scanning speed in 2PLM is limited by the long phosphorescence lifetime (microseconds). Nevertheless, point measurements in grid-like patterns ${ }^{114}$, which can be acquired rapidly, typically carry sufficient information about oxygen distributions as they inform about the state of the tissue metabolism.

Two-photon imaging of Absolute $\mathbf{p O}_{\mathbf{2}}$-Here we describe the steps necessary to implement 2PLM of resting state oxygen $\left(\mathrm{pO}_{2}\right)$ in cortical tissue in anesthetized mice using two-photon-enhanced phosphorescent nanoprobe PtP-C343 (Box 1) ${ }^{48}$. The experiment involves injection of PtP-C343 into the cortical tissue of the cranial window prior to permanently sealing of the window. A small amount of high molecular weight FITC-dextran $(2,000 \mathrm{kDa})$ is injected into the vasculature to aid in navigation and identification of imaging regions (Figure 8a,b). The fluorescein emission spectrum (green, 500-550 nm) does not overlap with phosphorescence of PtP-C343 (red-near IR, 650-800 nm), thus it can be used to map the vasculature without affecting oxygen measurements. We perform detection in two steps using a custom-built two-photon microscope and software equipped to excite and measure phosphorescence lifetimes ${ }^{114,120}$. First, we raster-scan the two-photon excitation beam over the field of view, rendering two-dimensional survey map images of the integrated emission intensity (Figure 8c). Survey images are used for both navigation within a cortical landscape and to assess the quality of the phosphorescence signal. Next, the PtP-C343 phosphorescence lifetime is imaged over a grid of points inside the cortical tissue (Figure $8 c)$. At each measurement location, we excite the phosphorescence by trains of femtosecond laser pulses, gated by an electro-optic modulator (EOM), and acquire phosphorescence decays by combining multiple excitation/detection cycles, typically about 500-2,000 decays (Figure 8d). Custom LabView software runs the microscope during the experiment. Afterwards, using Stern-Volmer calibration plots with parameters provided with each dye lot, we convert phosphorescence lifetimes, obtained by fitting the measured phosphorescent decays to single exponentials, into $\mathrm{pO}_{2}$ values (Figure 8e). The resulting $\mathrm{pO}_{2}$ data can be analyzed in multiple ways, from simple oxygenation histogram plots (Figure 8f) to more complex analyses.

While the fluorescein is sufficient to observe the vasculature during the phosphorescence experiment, the signal is not strong, and PtP-C343 contains coumarine-343 fragments, which exhibit green fluorescence and contribute to the background signal. Therefore, rhodamine $\mathrm{B}$, which fluoresces in the red with a peak near $610 \mathrm{~nm}$, is injected into the vasculature at the conclusion of the experiment to acquire background-free angiograms (Figure 8g,h). However, using rhodamine for blood plasma labeling during tissue $\mathrm{pO}_{2}$ imaging is not recommended, as its fluorescence may saturate the phosphorescence detector during the application of the phosphorescence excitation gate.

Additional technical considerations-Obtaining adequate SNR for fitting is one of the most important considerations for 2PLM. Phosphorescence intensity is typically low in the 
2PLM experiments - a condition that favors signal detection in photon counting mode with photomultiplier tube (PMT) detectors. In our setup, successful lifetime measurement of PtPC343 requires greater than a 10-fold ratio between the photon count rate at the end of the excitation gate (i.e. at the beginning of the phosphorescence decay) and the background signal at the end of the decay. Furthermore, it is important to use a PMT with significant sensitivity to PtP-C343 phosphorescence in the $650-800 \mathrm{~nm}$ range. For best SNR, the EOM used to gate the excitation light should have an extinction ratio (light transmission in "transparent" vs "opaque" mode) greater than 400. If other optical elements are used to gate the laser excitation instead of an EOM (e.g., acousto-optic deflector), they should exhibit a similarly large extinction ratio. Finally, using a long excitation wavelength $(\sim 920 \mathrm{~nm})$ outside the PMT detection range to excite phosphorescence eliminates the background signal due to the PMT exposure to the transmitted laser excitation light.

In this protocol, we used an objective heater to help maintain the brain surface at physiological temperature during imaging. It has been shown in mice that the brain surface temperature under the cranial window is lower than the expected $\sim 37^{\circ} \mathrm{C}^{121,122}$, which may influence the brain physiology. We include in the Materials section a list of the essential components that make up the custom 2PLM microscope, but refer to the detailed description of the construction and outfitting of the microscope system ${ }^{114,120}$ and PtP-C343 cortical dye injection method ${ }^{123}$ currently available in the literature.

Comparison to Alternative Methods-While other probes have been previously used for phosphorescent $\mathrm{pO}_{2}$ measurement, they often suffered from low signal and/or poor oxygen sensitivity ${ }^{124,125}$. In addition to the $\mathrm{pO}_{2}$ imaging technique we describe here, other tools for assessing tissue and intravascular oxygenation in cortex are oxygen electrodes, photoacoustic microscopy (PAM), and visible light OCT. Oxygen electrodes have long been the 'golden standard' of oximetry; however, these electrodes are invasive by nature, and their insertion inevitably inflicts tissue damage, thereby altering local physiology and oxygenation. Visible light OCT does not require exogenous contrast agent and it can quantify hemoglobin oxygen saturation in the pial vasculature, but it is not presently suitable for measurements in the capillaries and in the cortical depth in general. In addition, it cannot report cortical tissue $\mathrm{O}_{2}$ concentration. Similar to the absolute $\mathrm{pO}_{2}$ measurements, photoacoustic microscopy (PAM) can provide $\mathrm{O}_{2}$ saturation in all microvascular segments in 3D, and it has an advantage of relying on endogenous contrast (e.g. hemoglobin absorption). However, the PAM signal can be confounded by the changes in the tissue optical properties during measurement (e.g., $\mathrm{CBV}$ and/or $\mathrm{O}_{2}$ saturation changes) and the same endogenous contrast (hemoglobin absorption) cannot be utilized for tissue $\mathrm{pO}_{2}$ measurements.

\section{Cranial Window (steps 1-17)}

We present details of how we create agarose-sealed cranial windows. Here, the most important considerations are to keep brain surface blood vessels intact and maintain healthy brain tissue, which easily comes with some practice. There are many variations on cranial window technique, for example see ${ }^{123,126,127}$. Often the details depend on the location to be imaged, duration of imaging, and the type of head fixation used for the mouse. For most of the experiments described here, the mouse is held in a 6-axis stereotaxic frame throughout 
experiments, yielding open access to the top of the head. We open a cranial window over the hind limb somatosensory cortex for our experiments, but other regions such as whisker barrel cortex could be used depending on the purpose of the experiment and brain region of interest. Next, the area is covered with low-melt agarose solution and a glass coverslip is embedded into the agarose to seal the window (Figure 9). This allows the window and agarose to be peeled off and replaced between experiment steps if needed. Alternatively, a coverslip can be placed directly over the craniotomy and secured to the skull with cyanoacrylate glue or dental cement to create a permanent window. This variation is used for the absolute $\mathrm{pO}_{2}$ measurements, after injecting the PtP-C343 dye into the brain through the open window. Also for measurements of absolute $\mathrm{pO}_{2}$, the dura, a membranous layer that covers the brain, is removed from the window region to make dye injection easier.

Removing the dura is best accomplished immediately after opening the cranial window. We do not recommend removing the dura for the other techniques described here. There is a risk of damaging the brain when removing the dura, and the brain can swell a bit in the region where the dura is removed.

Comparison to Alternative Methods-To image the brain, we describe a technique to open a cranial window in the skull. A thinned skull preparation (e.g. ${ }^{128}$ ) is a potential alternative to a cranial window preparation. Because the skull is never actually opened, there is less chance of the procedure causing damage to the brain, edema, or gliosis. The drawback is that imaging depth achievable is typically more limited than for a craniotomy. For application of dyes such as SR101 or PtP-C343 for absolute tissue $\mathrm{pO}_{2}$ imaging, the challenge becomes how to administer the dyes into the brain and it may take some optimization to establish a reliable protocol.

\section{MATERIALS}

REAGENTS

Mice. We used 129S1/SvlmJ (Jackson Laboratory, 002448), platelet-derived growth factor receptor beta hemizygous (Pdgfr $\beta+/-$; provided by Dr. Philippe Soriano) ${ }^{129}$, and C57BL/6J (Charles River, strain code 27) mice in the examples shown.

CAUTION: Any experiments involving live mice must conform to relevant Institutional and National regulations. For the examples shown, procedures used were approved by the Institutional Animal Care and Use Committee at the University of Southern California and/or the Massachusetts General Hospital Subcommittee on Research Animal Care with National Institutes of Health guidelines.

Sterile aCSF (artificial cerebrospinal fluid), $\mathrm{pH} 7.4$, aliquoted in $1 \mathrm{~mL}$ volumes (Harvard Apparatus 59-7316)

Phospate-buffered saline, pH 7.4 (PBS; e.g. Sigma P3813, reconstituted according to manufacturer's instructions.)

Low-melt agarose (Sigma A9539)

Isotonic saline (Ricca 7210-16) 
D-glucose (Sigma G7021)

Fluorescein Isothiocyanate (FITC)-labeled Dextran $70 \mathrm{kDa}$ (ThermoFisher Scientific D1823)

FITC-Dextran, 2000kD (Sigma FD 2000S)

Sulforhodamine 101 (SR101) (ThermoFisher Scientific S359)

Rhodamine B Isothiocyanate-labeled Dextran 70 kDa (Rhodamine B; Sigma R9379)

PtP-C343 oxygen-sensitive dye (provided by Dr. Sergei A. Vinogradov) $)^{48}$

Cyanoacrilate gel glue, e.g. Loctite 454, 0.1 oz tubes (Amazon.com)

CRITICAL: Gel glue is highly recommended because its high viscosity helps keep the glue in place when applied.

Ortho-Jet liquid (Lang Dental Manufacturing)

CAUTION: OrthoJet liquid is a highly flammable liquid and vapor with a strong, unpleasant smell; it can cause skin irritation and an allergic skin reaction, as well as respiratory irritation. Careful handling is highly recommended.

Isoflurane, e.g. Fluriso (VetOne 502017)

Alpha-Chloralose (Sigma C0128)

Artificial Tears Ointment (Rugby 370435)

Nanofiltered water, e.g. from a Thermo Scientific Nanopure water filtration system (D11961) or similar

\section{EQUIPMENT}

Small Electric shaver

Surgery tools: Straight and $45^{\circ}$ forceps, fine tip $45^{\circ}$ forceps, scissors, microscalpel

Dissecting scope

Fine point permanent marker, e.g. Sharpie

Cotton tip (Q-tip) applicators

Micro or dental drill (11,000 rpm speed; CellPoint Scientific) with $0.6 \mathrm{~mm}$ burr (Fine Science Tools)

Air tubing connected to pressurized air source, such as lab air supply.

Gelfoam (Pfizer 09-0353-01) 
$3 \mathrm{~mm}$ or $5 \mathrm{~mm}$ diameter \#1 thickness glass coverslips (Warner Instruments 64-0720, 640700)

Stereotaxic frame, e.g. Kopf Instruments Model 1900, with large baseplate removed, and with mouse isoflurane mask and ear bars installed.

Hot plate with insert to hold $1.5 \mathrm{~mL}$ tubes.

Isoflurane vaporizer for anesthesia delivery (Kent Scientific SomnoSuite)

Electrical homeothermic heating pad with temperature regulation for rodents (e.g. 507053F, Harvard apparatus)

Pulse Oximeter ( $\mathrm{SpO}_{2}$ sensor; e.g. Kent Scientific MouseSTAT)

Mouse Ventilator (SAR-830, CWE Inc)

Blood Pressure Monitor (e.g. CWE Inc or Kent Scientific)

MicroCapnograph (Model 240, Columbus Instruments)

Insulin syringes for FITC-dextran dye injection, e.g. $1 \mathrm{~mL}, 12.7 \mathrm{~mm}$ length, $30 \mathrm{G}$ (BD 328411) work well for retro-orbital injection.

Small $0.2 \mu \mathrm{m}$ filters to filter glucose/saline solution.

Analog-to-digital converter (DAC), e.g. Molecular Devices DigiData 1550 Digitizer with accompanying software

Needle electrodes with wire leads and plugs to deliver electrical stimulus

Two-photon microscopes

Commercial systems: For diameter, velocity, and NADH imaging, use a two-photon microscope system with 20-25× 1 NA (or better) water immersion objective and appropriate emission filters for collecting the fluorescence signal of interest. Examples of suitable commercial systems include but are not limited to a Zeiss LSM 5 MP, Nikon A1R, and Bruker Nano Ultima or Investigator. Emission filters appropriate to detect the fluorescence of interest should be installed. For these systems, use the following filters, 500-550 nm band pass filter for FITC emission (Semrock); 435-485 nm band pass filter for NADH (Chroma or Zeiss); $650-710 \mathrm{~nm}$ band pass filter for SR101 (Chroma or Zeiss)

Custom made systems: For absolute pO2 imaging, one can use a customized twophoton microscope system fitted with phosporescence lifetime-based $\mathrm{pO}_{2}$ detectors and appropriate filters such as described by Sakadzic et al ${ }^{114}$. Examples of important components for this microscope include Femtosecond laser (InSight, Spectra Physics), Electro-Optic Modulator (EOM) and Driver (350-160/BK; 25D; Conoptics, Inc.), PhotonCounting Photomultiplier Tube (H10770PA-50, Hamamatsu), Phosphorescence emission filter (FF02-675/67-25, Semrock), CCD camera (CoolSnap FX, Roper Scientific), Low 
magnification objective (XLFLUOR 4X, Olympus), High magnification objective (XLUMPLFLN 20X, Olympus) and Flexible objective heater (TC-HLS-05, Bioscience Tools).

Commercial systems capable of these measurements, such as Bruker Nano Ultima and Investigator systems, are also now available.

$\mathrm{pO}_{2}$ dye injection equipment (following Goldey, et al ${ }^{123}$ )

Flaming/brown micropipette puller (Sutter Instruments, P-97) with $2.5 \mathrm{~mm}$ square box filament installed (Sutter Instruments, FB255B) or equivalent puller

Capillary glass, $1.0 \mathrm{~mm}$ outer diameter, $0.58 \mathrm{~mm}$ inner diameter (WPI, 1B100F-4)

Stereotaxic injector (Quintessential; Stoelting, 53311 or similar)

Hamilton syringe, 1.0 mm, $1701 \mathrm{RN}$, no needle (Hamilton, 7653-01)

Hamilton syringe priming kit (Hamilton, PRMKIT)

RN compression fitting $1 \mathrm{~mm}$ (Hamilton, 55750-01)

Light microscope (Olympus, BX50 or similar)

Reticle eyepiece for light microscope (Olympus, WH10X/22 or similar)

0.1-2.5 $\mu \mathrm{L}$ volume Pipettor (e.g. Eppendorf Research Plus; Fisher Scientific,

TI13-690-025) with gel loading tips (e.g. Eppendorf GELoader tips; Fisher Scientific, E5242956003)

MicroFil needle, 34 gauge, 67 mm long (WPI,. MF34G-5)

MicroFil needle, 28 gauge, $67 \mathrm{~mm}$ long (WPI, CMF28G)

IOS Imaging

IOS LEDs

Red IOS: $2 \times 3$ array of 6 high intensity $627 \mathrm{~nm}$ LEDs (Philips Lumileds Luxeon $\mathrm{K} 2$ ), spaced $2.54 \mathrm{~cm}$ apart, mounted on a holder that dissipates heat (heat sink), with wire leads to connect to power supply.

Green IOS: $2 \times 3$ array of 6 high intensity $530 \mathrm{~nm}$ LEDs (Philips Lumileds Luxeon K2), spaced $2.54 \mathrm{~cm}$ apart, mounted on a holder that dissipates heat (heat sink), with wire leads to connect to power supply.

Low electrical noise power supply to power LEDs (e.g. DC Regulated Power Supply, BK Precision 1670A)

IOS Filters

Red IOS: 640/40 nm (center wavelength/pass band) filter (Semrock FF01-640/40)

Green IOS: 522/36 nm filter (Chroma Technology Corp.)

Support and mounting hardware to hold LED array

Nat Protoc. Author manuscript; available in PMC 2019 March 06. 
Dissecting scope, e.g. Olympus SZ61. Optional: swing arm scope mount

High speed camera (e.g. MiCAM02-HR cMOS camera, SciMedia)

Vibration stimulus:

Vibration motor flat coin (Digi-Key 28821-ND)

$22 \mathrm{ohm} 1 \mathrm{~W}$ resistor

Connectors and wire to make connection to stimulus trigger, e.g. BNC connector and cable

Support to position and isolate stimulator and cable from table, e.g. Warner Instruments 69-0230

Electrical Stimulus:

Needle electrodes with wire leads

Current stimulus isolator, e.g. A385RC (WPI)

Software

Igor Pro 6 or higher (optional; WaveMetrics, https://www.wavemetrics.com/ index.html)

Matlab (MathWorks, https://www.mathworks.com/products/matlab.html),

Matlab with Biosignal-Specific Processing Pipeline software installed for $\mathrm{pO}_{2}$ measurements (https://www.mathworks.com/matlabcentral/fileexchange/59792-a-gui-onbiosignal-specific-processing-pipeline)

Matlab with line-scanning particle image velocimetry (LS-PIV) software installed for RBC velocity analysis ${ }^{41}$ (https://sourceforge.net/projects/lspivsupplement/files/)

IOS acquisition and analysis software (BV_Ana v.1604; SciMedia, http:// www.scimedia.com/fis/support/download/micam02/, or other)

Image $^{130} /$ FIJI (Fiji Is Just ImageJ) ${ }^{131}$ v. $1.51 \mathrm{~h}$ (https://fiji.sc/). FIJI is a version of imageJ that comes with many image analysis plug-ins preinstalled.

Labview (http://www.ni.com/en-us/shop/labview.html) with custom software routines for absolute pO2 measurement and phosphorescence lifetime fitting ${ }^{132}$ (Can be obtained by contacting Dr. Sakadžić or Dr. Vinogradov)

Computer with a multi-core processor (Intel i7 series or equivalent) for running data analysis

\section{REAGENT SETUP}

Agarose solution: Mix low-melt agarose with aCSF to yield a 2\% weight/volume solution. Heat the mixture on a hot plate to dissolve the agarose, mixing periodically until completely dissolved. Keep at $\sim 50^{\circ} \mathrm{C}$. The solution will solidify at room temperature $\left(\sim 22^{\circ} \mathrm{C}\right)$. The solution can be stored at $4^{\circ} \mathrm{C}$ for up to a week. 
Glucose/saline solution: Mix glucose into isotonic saline solution to yield a 5\% weight/ volume solution. Vortex well to dissolve. Filter with $0.2 \mu \mathrm{m}$ filter, and keep at room temperature up to 2 days. Can be kept at room temperature indefinitely if sterility is maintained. Can be made in large volumes, aliquoted $(0.5-1 \mathrm{~mL}$ volumes are often convenient) and frozen indefinitely until needed. Thaw to room temperature before use.

\section{Dyes:}

FITC-dextran, 70kDa: Reconstitute in isotonic saline to a concentration of $10 \mathrm{mg} / \mathrm{mL}$. Filter with a $0.2 \mu \mathrm{m}$ filter, aliquot and refrigerate $\left(4^{\circ} \mathrm{C}\right)$ or freeze $\left(-20^{\circ} \mathrm{C}\right)$ until ready to use. Dye will keep several weeks at $4^{\circ} \mathrm{C}$, and several months at $-20^{\circ} \mathrm{C}$. Protect from light.

SR 101: Reconstitute in aCSF to a concentration of $100 \mu \mathrm{M}$. Filter with a $0.2 \mu \mathrm{m}$ filter, aliquot $(0.5 \mathrm{~mL}$ volumes are often convenient $)$ and refrigerate $\left(4^{\circ} \mathrm{C}\right)$ or freeze $\left(-20^{\circ} \mathrm{C}\right)$ until ready to use. Dye will keep several months at either temperature. Protect from light.

Fluorescein isothiocyanate-Dextran, $2000 \mathrm{kDa}$ : Reconstitute at a concentration of 5\% weight/volume in PBS. Filter with a $0.2 \mu \mathrm{m}$ filter, aliquot $(0.5 \mathrm{~mL}$ volumes are often convenient) and freeze $\left(-20^{\circ} \mathrm{C}\right)$ until ready to use. Dye will keep several months at this temperature. Protect from light.

Rhodamine B: Reconstitute at a concentration of $2 \%$ weight/volume in PBS. Filter with a $0.2 \mu \mathrm{m}$ filter, aliquot $(0.5 \mathrm{~mL}$ volumes are often convenient) and freeze $\left(-20^{\circ} \mathrm{C}\right)$ until ready to use. Dye will keep several months at this temperature. Protect from light.

PtP-C343: Reconstitute in isotonic saline to a concentration of $140 \mu \mathrm{M}$. Filter with a $0.2 \mu \mathrm{m}$ filter, aliquot $\left(1 \mathrm{~mL}\right.$ volumes are often convenient) and freeze $\left(-20^{\circ} \mathrm{C}\right)$ until ready to use. Dye will keep several years at this temperature. Protect from light.

Gelfoam: Cut small 3-4 mm pieces of gelfoam and place in a $1.5 \mathrm{~mL}$ tube with room temperature aCSF. Allow to soak for at least $5 \mathrm{~min}$ before use. Typically $2-4$ pieces are needed per cranial window prep. Prepare pieces fresh for each cranial window procedure.

OrthoJet: Aliquot a small amount, $\sim 50-100 \mu \mathrm{L}$ into a $1.5 \mathrm{~mL}$ tube. Keep tube tightly closed until needed. Can be kept at room temperature indefinitely.

CAUTION: OrthoJet liquid is a highly flammable liquid and vapor with strong, unpleasant smell; it can cause skin irritation and an allergic skin reaction, as well as respiratory irritation. Careful handling is highly recommended.

\section{EQUIPMENT SETUP}

All imaging equipment should be set up on vibration isolation tables. Separate equipment that may cause vibration, optical, or electrical noise from the isolation table. See box 2 for details on how to set up the two-photon microscope for diameter, velocity, and NADH 
imaging. See box 3 for details on how to set up IOS equipment. See box 4 for details on how to set up Two-photon microscope for absolute tissue $\mathrm{pO}_{2}$ measurements

\section{PROCEDURE}

\section{Cranial window TIMING: Cranial window preparation takes about 30-60 min.}

CRITICAL: Inject mice with 5\% glucose in sterile saline ( $200 \mu \mathrm{L} / 25 \mathrm{~g}$ mouse)

Intraperitoneally (IP) every two hours while the animal is under anesthesia to maintain hydration.

1. Apply isoflurane anesthesia. Use $\sim 3 \%$ isoflurane to induce unconsciousness (usually requires about $2 \mathrm{~min}$ ), and maintain at $\sim 1 \%$ isoflurane. Position mouse in the stereotaxic frame. Ensure that the animal has achieved the proper depth of anesthesia. Anesthesia should be sufficient such that mouse does not respond to toe pinch. Maintain body temperature with electric heating pads and monitor respiration throughout procedure.

CAUTION: Procedures involving animals must be performed by qualified individuals according to institution and national vertebrate animal welfare guidelines.

2. Shave the top of the mouse's head and apply artificial tears ointment to the eyes.

3. Use forceps to raise the skin over approximate hind limb region of the somatosensory cortex (centered at $\mathrm{AP}=-1 \mathrm{~mm}, \mathrm{~L}=1.5 \mathrm{~mm}$ ) and cut open the skin. Push away the edges of the skin from the hind limb area.

4. Use a dry Q-tip to rub in a circular motion over the skull pushing away the connective tissue. Make sure the bone over the hind limb region is dry.

5. Depending on size of window desired, choose either $3 \mathrm{~mm}$ or $5 \mathrm{~mm}$ diameter coverslip for the window. Under a dissecting microscope, center the coverslip over the hind limb region ( $\mathrm{AP}=-1 \mathrm{~mm}, \mathrm{~L}=1.5 \mathrm{~mm}$ ). Use a fine tip permanent marker to draw a circle on the skull around the circumference of the coverslip. Set coverslip aside. For $3 \mathrm{~mm}$ coverslip windows, the location is easily found by aligning the coverslip edges along the midline and coronal sutures (near bregma). This corresponds to the center of the coverslip aligning atop the hind limb region of the somatosensory cortex.

6. Use micro drill (11,000 rpm speed) to drill along the inner perimeter of the marked circle. Use light, sweeping motions. Gently blow pressurized air across area while drilling to remove shavings and help keep skull cool. It is often simplest to hold the drill in one hand and the air tubing in the other. When the drill marks are visible, stop drilling and remove the marker using a Q-tip dipped in room temperature aCSF.

7. Dry skull thoroughly with a q-tip. Use cyanoacrylate glue to make a circular well around the cranial window area, about 2-3 mm larger in diameter then coverslip (Figure 9). 
8. Using a pipettor, add $10-20 \mu \mathrm{L}$ Orthojet liquid to the glue around the well to quickly dry the superglue. Try to apply Orthojet only to the glue, not the skull or other tissue. Gently blow pressured air over the glue to finish drying. When the superglue is completely dry it will be stiff when probed with a pipette tip.

CAUTION: Orthojet liquid is highly flammable liquid and vapor with strong, unpleasant smell; it can cause skin irritation and an allergic skin reaction, as well as respiratory irritation. Careful handling is highly recommended.

9. Continue drilling. Gently blow with air while drilling to get rid of skull dust. Apply room temperature aCSF frequently to help cool drilling site. Stop drilling when the drilled groves flex when the center skull plateau is lightly touched with the tip of the drill or forceps.

CAUTION: hold the drill at a very shallow angle and don't apply pressure; this prevents accidental penetration of the skull.

10. Add fresh aCSF to the well to cover. The drilled areas should look transparent. If not, remove aCSF and continue drilling. Then check flex (as described in step 9) and transparency again.

11. To lift off the bone from the window, place the tip of a microscalpel at the edge of the drilled grove in the window portion of the skull (almost parallel to the skull). Lodge the blade just enough into the bone to leverage. Use the raised superglue well as a fulcrum, and push the scalpel handle down lightly to gently lift up the bone in the window.

CAUTION: It is very important not to puncture the brain when lifting off the bone.

12. Use fine tip $45^{\circ}$ forceps to gently peel the piece of skull away from the brain and discard it. Immediately add aCSF to fill the well.

13. Add a piece of pre-soaked gelfoam over the window. The gelfoam will help stop bleeding from any surface, meningeal, or cranial vessels. If there is too much liquid in the well, dab the edge of the well lightly with a dry Q-tip to soak up some of the liquid.

14. Optional step: Dura membrane removal: Use a fine gauge syringe. With needle bevel side up, and needle almost parallel to the brain surface, brush the tip along the surface of the exposed brain until the tip catches the membrane. Gently pull the membrane away from the brain. Use fine tip $45^{\circ}$ forceps to help pull pieces of the dura membrane away from the brain.

CAUTION: Keep needle and forceps away from large vessels while removing dura. It is very easy to damage the brain surface during this procedure.

CRITICAL STEP: Perform dura removal as soon as possible after opening window. The dura becomes more difficult to remove the longer the window has been open.

15. Use forceps to gently move the gelfoam around in the well to absorb any blood. Replace with fresh gelfoam and rinse with aCSF frequently. 
16. When the bleeding has ceased, move the gelfoam around with a dry Q-tip to absorb most of the moisture. Immediately perform the next step.

CRITICAL STEP: Do not let the brain dry out.

17. Position coverslip and seal window. For IOS, NADH and two-photon diameter and velocity measurements follow option A for an agarose embedded coverslip to seal the window. For absolute $\mathrm{pO}_{2}$ measurements, follow option $\mathrm{B}$ for a cyanoacrilate gel glue-sealed window. The agarose method allows for easier removal and repositioning of the window.

A. Agarose embedded coverslip window.

i. Remove agarose from heat block $\left(\sim 50^{\circ} \mathrm{C}\right)$ and check temperature of agarose solution. Let it cool down for 1-2 min to approximately $40^{\circ} \mathrm{C}$. Touch to your skin to check temperature. Solution should feel warm but not hot. Could also check with a non-contact thermometer.

CRITICAL STEP: The agarose solution is kept at a temperature that is too high to apply directly to the brain. It must be allowed to cool down before applying to the brain.

ii. Draw $20-40 \mu \mathrm{L}$ of cooled $2 \%$ agarose solution into a pipet and apply to brain to cover the entire window.

iii. Using fine forceps, quickly embed the coverslip in the surface of the agarose, allowing the agarose to come just over the edge of the coverslip. Gently hold the coverslip in place with forceps and blow pressurized air across the area until the agarose solidifies. Add more agarose solution around the perimeter of the window to completely seal the coverslip if needed. Large surface vessels should be intact, and the window should be clear of any bleeding (see Figure 9 for example).

CRITICAL STEP: Avoid bubbles under the coverslip, and do not let the agarose completely cover the coverslip.

B. Cyanoacrilate gel glue-sealed coverslip window.

i. Position a glass coverslip over the open window. There should be just enough aCSF in the window to coat the side of the coverslip against the brain without any bubbles.

ii. Dry the skull around the window as much as possible with dry Q-tips. The glue will not hold well against wet surfaces.

iii. While holding the coverslip in place by gently pressing on the center with fine forceps, apply cyanoacrilate glue around the edges of coverslip and skull. 
CAUTION: Do not let the glue touch the brain surface. This could damage the brain, and cloud the window.

iv. Optional: Add small amounts of OrthoJet liquid to the glue surface to speed curing.

CRITICAL: Do not let the OrthoJet touch the brain surface. This will likely damage the brain.

PAUSE POINT: If a cyanoacrilate glue sealed window is placed, the mouse can be allowed to recover and returned to its home cage. Subsequent experiments can be resumed 7-10 days or more later. For experiments with agarose sealed windows and same-day experiments with cyanoacrilate glue sealed windows, maintain anesthesia and temperature regulation, do not pause.

18. Perform imaging and analysis. Follow option A to perform intrinsic optical signal imaging of regional hemodynamic responses and oxygen delivery to brain. Follow option B to perform high-resolution two-photon microscopy microvascular diameter and velocity measurements. Follow option $\mathrm{C}$ to perform NADH two-photon imaging and measurements. Follow Box 5 for instructions on the Mouse setup for two-photon diameter, RBC velocity, or NADH imaging and Box 1 to perform two-photon imaging of absolute $\mathrm{pO}_{2}$ (See figure 1.)

\section{A. IOS imaging of regional hemodynamic or oxygen delivery responses}

TIMING: This process should take 30-60 min for short stimulus paradigms, and about 60 $90 \mathrm{~min}$ for long stimulus paradigms. Image processing takes 15-60 minutes per mouse.

i. Position anesthetized mouse in stereotaxic frame under dissecting scope.

ii. Position heating pad and thermal probe to maintain mouse's body temperature.

iii. Affix electrical stimulus or mechanical stimulus to the contralateral hind limb. If using an electrical stimulus insert electrical probes (needles) at top (near toes) and base of foot pad. Adjust the current amplitude such that the animal receives the stimulus but does not feel pain (i.e. gets startled). Do this by observing the paw with the electrodes while applying a stimulus large enough to produce twitching easily observable by eye, then gradually decrease the amplitude until twitching is barely visible by eye. We call this the 'visual threshold'. Typical currents are in $1 \mathrm{~mA}$ range. If using a mechanical stimulus affix a vibrating motor to the foot pad using a narrow strip of lab tape wrapped around foot and motor. Tape should be tight enough to secure motor against foot, but not so tight as to be uncomfortable or cause circulation issues. Adjust or elevate foot slightly so that it does not touch anything connected to the stereotaxic frame. This minimizes artifacts from the vibrations during stimulus.

iv. Visually confirm that mouse does not show movement in response to stimulus. Mouse should not startle or retract foot in response to stimulus.

v. Use either white light or LED illumination to focus on the surface of the brain. Adjust stereotaxic frame to make window as level as possible relative to the 
scope. Zoom to desired magnification. Typical zoom in our system is $\sim 4 \mathrm{X}$. Let the LEDs warm up a couple minutes before recording to ensure more stable illumination.

CAUTION: Follow all safety procedures for LED operation.

vi. Focus slightly below surface of the brain.

vii. Set cMOS camera exposure to $30 \mathrm{~ms}$. Choose either short or long stimulus protocol. The short protocol is often adequate for observing differences between conditions or locating the region responsive to stimulus. The long protocol is better if matching stimulus parameters to that used in other experiments such as diameter, velocity, and NADH measurements is required. For the short stimulus acquire 40 s-long inter-stimulation interval (ISI) consisting of 15 s-long recording and 25-s-long delay for data saving and blood flow to return to baseline. We acquired and averaged 10 ISIs. Set 300-ms-long stimulus $(10 \mathrm{~Hz}$ if using electrical stimulus) to start at least $1 \mathrm{~s}$ into recording for recording of the baseline signal. For long stimulus acquire 110 s-long ISI consisting of 40-s-long recording and 70 s-long delay for data saving and blood flow to return to baseline. We acquired and averaged 10 ISIs. Set 10 s-long stimulus $(10 \mathrm{~Hz}$ if using electrical stimulus) to start $5 \mathrm{~s}$ into recording.

viii. Acquire data.

CRITICAL STEP For best results, room should be dark.

?Troubleshooting

ix. If subsequent imaging experiments are planned for the mouse that rely on IOS imaging (e.g. diameter, velocity, or NADH measurements), only perform IOS image processing steps 18A.x-xii below to identify activation center. Mouse can be maintained under anesthesia, with temperature regulation, on the stereotaxic frame during this time. Full IOS image analysis can be performed at a later time. Otherwise once data acquisition is complete, mouse can either be sacrificed in accordance with Institutional and National regulations, or a glue sealed cranial window can be installed (step 17B) and the mouse allowed to recover and return to its home cage.

PAUSE POINT: IOS image processing can be performed at a later day or time if further experiments planned for the animal do not rely on IOS signal location information.

CAUTION Ensure that experiments involving live animals conform to Institutional and National regulations.

IOS image processing (TIMING: 15-60 min per mouse) Average 10 recorded ISIs. CRITICAL Image processing is performed with the semi-automated routines in the BV_ANA software, or similar software package. Subsequent analysis is performed on a computed mean ISI sequence. 
x. Find the location of the activation center by identifying the location of the maximum response to the stimulus. Compute a baseline frame by averaging the frames before stimulus. Compute activation frame by averaging $1 \mathrm{~s}$-long interval at the beginning of the stimulus. This early response is typically better correlated with the activation center, while the signal later on could be more affected by the hemodynamic response spread out by the pial vasculature over a larger cortical area.

xi. Compute a ratio of the activation frame to the baseline frame. The activation center colocalizes with the signal minimum in the computed frame.

xii. Compute a mean time course of the relative IOS change at the activation center. Select an ROI within the activation center, typically $\sim 200 \mu \mathrm{m}$ across and away from large pial vessels. Average the pixels in the ROI for each frame in the mean ISI sequence to obtain a mean signal from the activation center. Compute the baseline ROI signal value by averaging the mean signal before stimulus. Normalize the mean ROI signal by subtracting the baseline ROI signal and subsequently dividing the result by the same baseline signal. This yields the mean reflected light intensity change due to stimulus $(\Delta \mathrm{I}$, where $\mathrm{I}$ is intensity) over time within the activation center ROI, as a fraction of the baseline intensity $\left(\mathrm{I}_{\mathrm{O}}\right)$. If needed, a low-pass temporal filter $(0.5-1.0 \mathrm{~Hz})$ may be applied to the result to further suppress the noise.

\section{B. Two-photon imaging of microvascular diameter and velocity measurements}

TIMING: Two-photon imaging of microvascular diameter and RBC velocity typically takes about 2-4 hours. The analysis for capillary diameter and RBC velocity takes 1 day per mouse

i. $\quad$ Prepare the mice as described in box 5

ii. Prepare two-photon microscope for imaging FITC fluorescence: Tune laser to wavelength between $800-820 \mathrm{~nm}$ excitation, and set emission filters to $500-550$ nm.

iii. Inject $100 \mu \mathrm{L}$ of prepared FITC-dextran $(70 \mathrm{kDa})$ dye solution via retro-orbital injection (or tail vein injection).

iv. Within the activation center, identify arterioles based on morphology and/or blood flow direction based on RBC movement using either widefield fluorescence of blood plasma (RBCs appear dark in dye-loaded vasculature) or visualization in white light reflected from the brain surface with widefield imaging.

v. Switch to two-photon excitation and follow arteriole descending into the cortex. Identify locations for diameter and velocity measurements on arterioles and capillary branches.

vi. Diameter measurements: After selecting a location to measure vessel diameter, use live imaging microscope mode to adjust the focal plane to cross the vessel 
through its center, such that the vascular diameter is maximal. Set up a line scan perpendicular to the axis of the vessel.

vii. Set linescan pixel resolution of $20-50 \mathrm{~nm} /$ pixel, with 512 pixels per line. Set the linescan to average multiple unidirectional lines per time point, resulting in a net scanning rate of $15 \mathrm{~Hz}$ (15 averaged lines per second) or better. Typical settings would be to average 8 lines per time point, resulting in a net $15 \mathrm{~Hz}$ linescan acquisition. This should be achievable in both commercial and custom-built twophoton microscopes, which are generally capable of supporting linescan rates well in excess of $100 \mathrm{~Hz}$ with pixel dwell times on the order of a few $\mu$ s.

viii. Set the Inter-Stimulation Interval (ISI) to $90 \mathrm{~s}$, consisting of 20-s-long baseline, 10 s-long stimulus $(10 \mathrm{~Hz})$ and 60 s-long post-stimulus recovery. Acquire the linescans throughout entire duration of ISIs.

ix. RBC velocity measurements: At the same location selected for the vessel diameter measurement, set up a linescan along the vessel axis.

$\mathbf{x}$. Set the line scan rate to the maximum possible, bidirectional scanning, no line averaging. Typical settings were 256 pixels per line with $120 \mathrm{~nm} / \mathrm{pixel}$, and linescan rate of $2.6 \mathrm{kHz}$.

xi. Set the ISI to $90 \mathrm{~s}$, consisting of 20-s-long baseline, 10 s-long stimulus $(10 \mathrm{~Hz})$ and 60 s-long post-stimulus recovery. Acquire the linescans throughout entire duration of ISIs.

CAUTION: at this scan rate, the number of lines scanned during a measurement may be limited by the acquisition computer's memory, at least in older microscope systems with more limited memory.

xii. Once data acquisition is complete, mouse can either be sacrificed in accordance with Institutional and National regulations, or a glue-sealed cranial window can be installed (step 17B) and the mouse allowed to recover and return to its home cage.

PAUSE POINT: Diameter and velocity analysis can be performed at a later day or time, after the imaging experiments are complete.

xiii. Diameter analysis: Load linescan image (linescans recorded over time built up into an image, or kymograph) into analysis software such as Igor Pro, and smooth with a 3 pixel (sigma $=3$ ) 2D Gaussian filter.

xiv. Threshold the image using the "fuzzy entropy" setting. This sets thresholds based on Shannon's Entropy function ${ }^{94}$. Pixels making up the vessel become white, and the background black.

xv. Determine the vessel diameter at each line in the kymograph by measuring the number of white pixels in each line. This results in a measure of diameter, in pixels, vs. time. 
xvi. Remove heartbeat and breathing artifacts from the data trace by low-pass filtering with a Kaiser Bessel filter with a $1 \mathrm{~Hz}$ or more cut off, and notch filter set to $\geq 1 \mathrm{~Hz}$.

CRITICAL STEP: The notch filter removes most breathing related artifacts and should be typically set to $1 \mathrm{~Hz}$ or more with $0.5 \mathrm{~Hz}$ bandwidth. However, optimal notch filter frequency may vary slightly by animal and from measurement to measurement. Inspect the individual traces to ensure that for both filters the correct frequency was filtered out.

xvii. Filter the resulting data with a sliding box filter with a $1 \mathrm{~s}$ window. Optional: Data trace can be normalized to its basal value.

xviii. Traces should be inspected for noise. Traces that show a drift in the baseline diameter (before stimulus) should be excluded from subsequent analysis. This drift indicates that the vessel is shifting its position relative to the focal plane. Traces with large variation should be excluded from subsequent analysis as well. In our hands in diameter traces with average baseline normalized to 1 , a noisy signal may have a variance on the order of $10^{-3}$ measured during the basline.

xix. To determine time to 50\% peak diameter, fit a sigmoid curve to the diameter increase portion of the trace. The fit parameters will yield a 50 max parameter.

xx. Velocity analysis: Using the Matlab "LS-PIV" algorithm ${ }^{41}$, process RBC flow linescan image (linescans recorded over time built up into an image, or kymograph). The output will be a trace of RBC velocity over time. Save the data to a spreadsheet. For our image settings, LS-PIV parameters used were: number of averages (numavgs) $=200$, skip amount $($ skipamt $)=25$, and shift amount $($ shiftamt $)=1$.

xxi. Plot the velocity trace to check for individual extremely large or small outlier points, $3 \mathrm{SD}$ or more from the mean of the trace. Replace any outliers found with the median of the 25 points surrounding the outlier point, centered on that point.

xxii. Remove heartbeat and breathing artifacts from the data trace by low-pass filtering with a Kaiser Bessel filter with a $1 \mathrm{~Hz}$ or more cut off, and notch filter set to $\geq 1 \mathrm{~Hz}$.

CRITICAL STEP: The notch filter does the bulk of filtering out the breathing artifact, and is typically set to $1 \mathrm{~Hz}$ or more with $0.5 \mathrm{~Hz}$ bandwidth, but this can vary by animal and from measurement to measurement. Inspection of the individual traces to ensure for both filters that the correct frequency was filtered out is necessary.

xxiii. Filter the resulting data with a sliding box filter with a $1 \mathrm{~s}$ window. This filter takes each point and replaces it with the average of the points in the filter window, centered on that point. Optional: Data trace can be normalized to its basal value.

xxiv. To determine time to $50 \%$ peak diameter, fit a sigmoid curve to the diameter increase portion of the trace. The fit parameters will yield a 50 max parameter. 


\section{NADH imaging}

TIMING: NADH imaging typically takes about $30 \mathrm{~min}$ and image analysis takes 1-2 hours per mouse

CRITICAL Maintain mouse under anesthesia with appropriate monitoring for the following steps.

i. Perform IOS measurement to identify the activation center (Step 18A or Box 5 step 1).

ii. Under a dissecting microscope, with mouse maintained under anesthesia with temperature regulation on the stereotaxic frame, carefully peel away agarose and coverslip from the craniotomy with fine tweezers. Rinse once and keep moist with aCSF.

iii. Carefully draw off excess aCSF from the brain surface and add SR101 dye solution to the open cranial window. Add enough liquid to make a prominent "bubble" over the window. Cover mouse with foil to protect from light, and allow SR101 uptake by astrocytes for 15 minutes.

iv. While dye is incubating, prepare two-photon microscope for imaging: Tune laser to $740 \mathrm{~nm}$ excitation, and set filters to collect fluorescence emission in blue (435-485 nm; NADH) and red (650-710 nm; SR101).

v. Remove dye solution and wash with aCSF. Seal the window with agarose and coverslip as described above (step 17A).

vi. Prepare mouse for two-photon imaging by following Box 5 steps 2-5.

vii. Identify the active brain region from the IOS measurement, and focus down from the surface to the desired measurement depth. Typical imaging depths are 50$150 \mu \mathrm{m}$-- use the same depth for all animals in the study. Try to avoid large surface vessels.

viii. Set the ISI to $90 \mathrm{~s}$, consisting of 20 -s-long baseline, $10 \mathrm{~s}$-long stimulus $(10 \mathrm{~Hz})$ and 60 s-long post-stimulus recovery. Acquire the image frames throughout entire duration of ISIs, interleaving NADH and SR101 image acquisitions. In our system, we needed $6 \mathrm{~s}$ to acquire an interleaved NADH/SR101 pair, with 411 $\mathrm{nm} /$ pixel resolution $(210 \times 210 \mathrm{um}$ area) and four (bidirectional) scans averaged together to generate each image

CRITICAL STEP: Excessive excitation laser power can cause NADH bleaching, which can be identified by both inspecting the NADH fluorescence intensity change in time (e.g., exhibits exponential decay) and by increasing the field of view (e.g., previous field of view appears as a dark square). We have observed that even lower excitation powers, which do not produce noticeable bleaching, can still have adverse effect on the NADH signal showing an initial increase of the baseline NADH fluorescence intensity over the course of few minutes from the beginning of the laser exposure. Both conditions should be avoided by sufficiently tuning down the laser excitation power. 
ix. Once data acquisition is complete, mouse can either be sacrificed in accordance with Institutional and National regulations, or a glue-sealed cranial window can be installed (step 17B) and the mouse allowed to recover and return to its home cage.

CAUTION Ensure that experiments involving live animals conform to Institutional and National regulations.

PAUSE POINT: NADH image analysis can be performed at a later date or time, after the imaging experiments are complete.

x. NADH image analysis: Open NADH and corresponding SR101 image sets in ImageJ.

xi. Draw an ROI on the NADH image sequence to encompass as much of the image field as possible, excluding edges of the image and locations where there are shadows of large vessels. Save a copy of the ROI to the ROI manager.

?Troubleshooting

xii. Use ImageJ's measurement commands to measure the average ROI intensity for each image in the NADH sequence. The resulting data is the average intensity of the ROI over the time course of the image sequence. Save to a spreadsheet or desired program.

xiii. Apply the NADH ROI from step 18C.xi to the SR101 image set, and perform analysis as in the previous step (step 18C.xii) for the SR101 data.

xiv. Normalize the time courses for the NADH and SR101 traces to the averages of their respective baselines for the $10 \mathrm{~s}$ preceding stimulus.

xv. Multiply the SR101 ROI data trace by 1.15, and subtract the resulting SR101 trace from the NADH trace. This yields the corrected NADH time course trace. The scaling factor was determined by previous numerical simulations and confirmed with experimental data 105

\section{TIMING}

Two-photon set up for diameter, velocity, and NADH imaging takes about 20 min (Box 2).

IOS setup takes $1-2 \mathrm{hr}$ for initial equipment and software setup, and $\sim 10$ min subsequently (Box 3).

Two-photon set up for absolute tissue $\mathrm{pO}_{2}$ measurements takes about 30 min (Box 4).

Cranial window preparation takes about 30-60 min (steps 1-17).

IOS imaging should take 30-60 min for short stimulus paradigms, and about 60-90 min for long stimulus paradigms (steps 18A.i-viii).

IOS image analysis should take about 15-60 min per mouse (Steps 18A.ix-xiii) 
Mouse setup for two-photon diameter, RBC velocity, or NADH imaging takes about 10-20 $\min ($ Box 5)

Two-photon imaging of diameter and velocity typically takes about $2-4$ hours (steps 18B.ixii).

Analysis of two-photon imaging of diameter and velocity typically takes about 1 day per mouse (Steps 18B.xiii-xxiv)

NADH imaging typically takes about 30 min (steps 18C.i-ix).

Analysis of NADH imaging typically takes about 1-2 hours per mouse (18C.x-xv)

Absolute $\mathrm{pO}_{2}$ measurements take about 2 hrs, after a 2 hour prep (Box 1 steps 1-19).

Analysis of Absolute $\mathrm{pO}_{2}$ measurements take about 2 hours per mouse (Box 1 steps 20-22).

\section{ANTICIPATED RESULTS}

With these techniques, one can discern vascular function and tissue oxygenation on the regional to local level. All techniques require mastering a cranial window preparation (Figure 9), which can be achieved by any competent graduate student or research staff. With high resolution two-photon linescans, the researcher can measure small changes in capillary or arteriole diameter (Figure 2). Similarly, RBC velocity changes can be measured in these same vessels (Figure 3 ). Velocity can vary by vessel size and branch order. With a relatively simple setup (Figure 6), IOS imaging provides regional information about changes in hemodynamic responses (with green light; Figure 4) and oxyhemoglobin changes reflecting for the most part changes in $\mathrm{O}_{2}$ delivery vs demand (with red light; Figure 5) in response to stimulus. Two-photon NADH imaging reveals the balance between tissue $\mathrm{O}_{2}$ supply and demand. While the corrected NADH signal change in response to relatively short (10 s) stimulus is typically unremarkable in healthy mouse brains, it can be significant in animals with disrupted neurovascular coupling, with more positive corrected NADH values correlating to more reduced tissue oxygenation (Figure 7). And, for more localized measurement of $\mathrm{O}_{2}$ partial pressure, two-photon imaging of phosphorescent $\mathrm{pO}_{2}$ sensitive dye reveals information about local differences in tissue oxygenation (Figure 8). In combination with microvascular angiograms (Figure 8g,h), measured tissue $\mathrm{pO}_{2}$ values can be used to compute oxygen concentration distributions as a function of distances from arterioles, venules, and capillaries.

\section{Supplementary Material}

Refer to Web version on PubMed Central for supplementary material.

\section{ACKNOWLEDGMENTS}

The work is supported by the US National Institutes of Health grants R01AG023084, R01NS090904, R01NS034467, R01AG039452, R01NS100459, P01AG052350 to B.V.Z., R24NS092986, R01EB018464, and R01NS091230 to S.S., S.A.V. and D.A.B., Alzheimer's Association and Cure Alzheimer's fund to B.V.Z, and Fondation Leducq Transatlantic Network of Excellence for the Study of Perivascular Spaces in Small Vessel 
Disease, ref no. 16 CVD 05 to B.V.Z. We thank R. Jaswal for helping make figure 8 . We gratefully acknowledge the feedback, forum posts, and questions from our peers regarding the techniques presented here, who provided the inspiration for this manuscript.

\section{REFERENCES}

1. Kety SS THE GENERAL METABOLISM OF THE BRAIN IN VIVO in Metabolism of the Nervous System 221-237 (Elsevier, 1957).

2. Sokoloff $\mathrm{L}$ The metabolism of the central nervous system in vivo in Handbook of Physiology, Section I, Neurophysiology (eds. Field J, Magoun HW \& Hall VE) 3, 1843-1864 (American Physiological Society, 1960).

3. Zlokovic BV Neurovascular pathways to neurodegeneration in Alzheimer's disease and other disorders. Nat. Rev. Neurosci 12, 723-738 (2011). [PubMed: 22048062]

4. Sweeney MD, Sagare AP \& Zlokovic BV Blood-brain barrier breakdown in Alzheimer disease and other neurodegenerative disorders. Nat. Rev. Neurol (2018). doi:10.1038/nrneurol.2017.188

5. Iadecola C The pathobiology of vascular dementia. Neuron 80, 844-866 (2013). [PubMed: 24267647]

6. Kisler K, Nelson AR, Montagne A \& Zlokovic BV Cerebral blood flow regulation and neurovascular dysfunction in Alzheimer disease. Nat. Rev. Neurosci (2017). doi:10.1038/nrn. 2017.48

7. Arvanitakis Z, Capuano AW, Leurgans SE, Bennett DA \& Schneider JA Relation of cerebral vessel disease to Alzheimer's disease dementia and cognitive function in elderly people: a cross-sectional study. Lancet Neurol. 15, 934-943 (2016). [PubMed: 27312738]

8. Iturria-Medina Y et al. Early role of vascular dysregulation on late-onset Alzheimer's disease based on multifactorial data-driven analysis. Nat. Commun 7, 11934(2016). [PubMed: 27327500]

9. Montagne A et al. Brain imaging of neurovascular dysfunction in Alzheimer's disease. Acta Neuropathol. (Berl.) 131, 687-707 (2016). [PubMed: 27038189]

10. Montagne A, Zhao Z \& Zlokovic BV Alzheimer's disease: A matter of blood-brain barrier dysfunction? J. Exp. Med jem.20171406 (2017). doi:10.1084/jem.20171406

11. Murphy MJ et al. Widespread cerebral haemodynamics disturbances occur early in amyotrophic lateral sclerosis. Amyotroph. Lateral Scler. Off. Publ. World Fed. Neurol. Res. Group Mot. Neuron Dis 13, 202-209 (2012).

12. Ishikawa T, Morita M \& Nakano I Constant blood flow reduction in premotor frontal lobe regions in ALS with dementia - a SPECT study with 3D-SSP. Acta Neurol. Scand 116, 340-344 (2007). [PubMed: 17922728]

13. Yamashita $\mathrm{T}$ et al. Flow-metabolism uncoupling in the cervical spinal cord of ALS patients. Neurol. Sci. Off. J. Ital. Neurol. Soc. Ital. Soc. Clin. Neurophysiol 38, 659-665 (2017).

14. Verfaillie SCJ et al. Cerebral perfusion and glucose metabolism in Alzheimer's disease and frontotemporal dementia: two sides of the same coin? Eur. Radiol 25, 3050-3059 (2015). [PubMed: 25899416]

15. Malek $\mathrm{N}$ et al. Vascular disease and vascular risk factors in relation to motor features and cognition in early Parkinson's disease. Mov. Disord. Off. J. Mov. Disord. Soc 31, 1518-1526 (2016).

16. Al-Bachari S, Vidyasagar R, Emsley HC \& Parkes LM Structural and physiological neurovascular changes in idiopathic Parkinson's disease and its clinical phenotypes. J. Cereb. Blood Flow Metab. Off. J. Int. Soc. Cereb. Blood Flow Metab $271678 X 16688919$ (2017). doi: $10.1177 / 0271678 X 16688919$

17. Drouin-Ouellet J et al. Cerebrovascular and blood-brain barrier impairments in Huntington's disease: Potential implications for its pathophysiology. Ann. Neurol 78, 160-177 (2015). [PubMed: 25866151]

18. Chen JJ, Salat DH \& Rosas HD Complex relationships between cerebral blood flow and brain atrophy in early Huntington's disease. NeuroImage 59, 1043-1051 (2012). [PubMed: 21945790]

19. Wardlaw JM, Smith C \& Dichgans M Mechanisms of sporadic cerebral small vessel disease: insights from neuroimaging. Lancet Neurol. 12, 483-497 (2013). [PubMed: 23602162] 
20. Wardlaw JM et al. Neuroimaging standards for research into small vessel disease and its contribution to ageing and neurodegeneration. Lancet Neurol. 12, 822-838 (2013). [PubMed: 23867200]

21. Montine TJ et al. Recommendations of the Alzheimer's disease-related dementias conference. Neurology 83, 851-860 (2014). [PubMed: 25080517]

22. Snyder HM et al. Vascular contributions to cognitive impairment and dementia including Alzheimer's disease. Alzheimers Dement. J. Alzheimers Assoc 11, 710-717 (2015).

23. Hachinski V \& World Stroke Organization. Stroke and Potentially Preventable Dementias Proclamation: Updated World Stroke Day Proclamation. Stroke 46, 3039-3040 (2015). [PubMed: 26504189]

24. Faraco G \& Iadecola C Hypertension: a harbinger of stroke and dementia. Hypertens. Dallas Tex 1979 62, 810-817 (2013).

25. Last D et al. Global and regional effects of type 2 diabetes on brain tissue volumes and cerebral vasoreactivity. Diabetes Care 30, 1193-1199 (2007). [PubMed: 17290035]

26. Ingrisch $M$ et al. Quantification of perfusion and permeability in multiple sclerosis: dynamic contrast-enhanced MRI in 3D at 3T. Invest. Radiol 47, 252-258 (2012). [PubMed: 22373532]

27. De Roos A, van der Grond J, Mitchell G \& Westenberg J Magnetic Resonance Imaging of Cardiovascular Function and the Brain: Is Dementia a Cardiovascular-Driven Disease? Circulation 135, 2178-2195 (2017). [PubMed: 28559496]

28. Qiu C \& Fratiglioni L A major role for cardiovascular burden in age-related cognitive decline. Nat. Rev. Cardiol 12, 267-277 (2015). [PubMed: 25583619]

29. Lok J et al. Targeting the neurovascular unit in brain trauma. CNS Neurosci. Ther 21, 304-308 (2015). [PubMed: 25475543]

30. Toth $\mathrm{P}$ et al. Traumatic brain injury-induced autoregulatory dysfunction and spreading depressionrelated neurovascular uncoupling: Pathomechanisms, perspectives, and therapeutic implications. Am. J. Physiol. Heart Circ. Physiol 311, H1118-H1131 (2016). [PubMed: 27614225]

31. Najjar S et al. Neurovascular Unit Dysfunction and Blood-Brain Barrier Hyperpermeability Contribute to Schizophrenia Neurobiology: A Theoretical Integration of Clinical and Experimental Evidence. Front. Psychiatry 8, 83(2017). [PubMed: 28588507]

32. Ances B, Vaida F, Ellis R \& Buxton R Test-retest stability of calibrated BOLD-fMRI in HIV- and HIV+ subjects. NeuroImage 54, 2156-2162 (2011). [PubMed: 20932922]

33. Iadecola $\mathrm{C}$ et al. SOD1 rescues cerebral endothelial dysfunction in mice overexpressing amyloid precursor protein. Nat. Neurosci 2, 157-161 (1999). [PubMed: 10195200]

34. Niwa K et al. Abeta 1-40-related reduction in functional hyperemia in mouse neocortex during somatosensory activation. Proc. Natl. Acad. Sci. U. S. A 97, 9735-9740 (2000). [PubMed: 10944232]

35. Chow $\mathrm{N}$ et al. Serum response factor and myocardin mediate arterial hypercontractility and cerebral blood flow dysregulation in Alzheimer's phenotype. Proc. Natl. Acad. Sci. U. S. A 104, 823-828 (2007). [PubMed: 17215356]

36. Miyazaki K et al. Early and Progressive Impairment of Spinal Blood Flow-Glucose Metabolism Coupling in Motor Neuron Degeneration of ALS Model Mice. J. Cereb. Blood Flow Metab 32, 456-467 (2012). [PubMed: 22068226]

37. Kleinberger $\mathrm{G}$ et al. The FTD-like syndrome causing TREM2 T66M mutation impairs microglia function, brain perfusion, and glucose metabolism. EMBO J. 36, 1837-1853 (2017). [PubMed: 28559417]

38. Toth $\mathrm{P}$ et al. Age-related autoregulatory dysfunction and cerebromicrovascular injury in mice with angiotensin II-induced hypertension. J. Cereb. Blood Flow Metab. Off. J. Int. Soc. Cereb. Blood Flow Metab 33, 1732-1742 (2013).

39. Faraco $\mathrm{G}$ et al. Hypertension enhances $\mathrm{A} \beta$-induced neurovascular dysfunction, promotes $\beta$ secretase activity, and leads to amyloidogenic processing of APP. J. Cereb. Blood Flow Metab. Off. J. Int. Soc. Cereb. Blood Flow Metab 36, 241-252 (2016).

40. Kisler K et al. Pericyte degeneration leads to neurovascular uncoupling and limits oxygen supply to brain. Nat. Neurosci 20, 406-416 (2017). [PubMed: 28135240] 
41. Kim TN et al. Line-scanning particle image velocimetry: an optical approach for quantifying a wide range of blood flow speeds in live animals. PloS One 7, e38590(2012). [PubMed: 22761686]

42. Harrison TC, Sigler A \& Murphy TH Simple and cost-effective hardware and software for functional brain mapping using intrinsic optical signal imaging. J. Neurosci. Methods 182, 211218 (2009). [PubMed: 19559049]

43. Hillman EMC Optical brain imaging in vivo: techniques and applications from animal to man. J. Biomed. Opt 12, 051402(2007). [PubMed: 17994863]

44. Sirotin YB, Hillman EMC, Bordier C \& Das A Spatiotemporal precision and hemodynamic mechanism of optical point spreads in alert primates. Proc. Natl. Acad. Sci. U. S. A 106, 18390 18395 (2009). [PubMed: 19828443]

45. Frostig RD, Lieke EE, Ts'o DY \& Grinvald A Cortical functional architecture and local coupling between neuronal activity and the microcirculation revealed by in vivo high-resolution optical imaging of intrinsic signals. Proc. Natl. Acad. Sci 87, 6082-6086 (1990). [PubMed: 2117272]

46. Kasischke KA, Vishwasrao HD, Fisher PJ, Zipfel WR \& Webb WW Neural activity triggers neuronal oxidative metabolism followed by astrocytic glycolysis. Science 305, 99-103 (2004). [PubMed: 15232110]

47. Mayevsky A \& Rogatsky GG Mitochondrial function in vivo evaluated by NADH fluorescence: from animal models to human studies. AJP Cell Physiol. 292, C615-C640 (2006).

48. Finikova OS et al. Oxygen microscopy by two-photon-excited phosphorescence. Chemphyschem Eur. J. Chem. Phys. Phys. Chem 9, 1673-1679 (2008).

49. Gama Sosa MA et al. Age-Related Vascular Pathology in Transgenic Mice Expressing Presenilin 1-Associated Familial Alzheimer's Disease Mutations. Am. J. Pathol 176, 353-368 (2010). [PubMed: 20008141]

50. Blair LJ et al. Tau depletion prevents progressive blood-brain barrier damage in a mouse model of tauopathy. Acta Neuropathol. Commun 3, (2015).

51. Lewis $\mathbf{J}$ et al. Neurofibrillary tangles, amyotrophy and progressive motor disturbance in mice expressing mutant (P301L) tau protein. Nat. Genet 25, 402-405 (2000). [PubMed: 10932182]

52. Bell RD et al. Apolipoprotein E controls cerebrovascular integrity via cyclophilin A. Nature 485, 512-516 (2012). [PubMed: 22622580]

53. Alata W, Ye Y, St-Amour I, Vandal M \& Calon F Human apolipoprotein E $\varepsilon 4$ expression impairs cerebral vascularization and blood-brain barrier function in mice. J. Cereb. Blood Flow Metab. Off. J. Int. Soc. Cereb. Blood Flow Metab 35, 86-94 (2015).

54. Nelson AR, Sweeney MD, Sagare AP \& Zlokovic BV Neurovascular dysfunction and neurodegeneration in dementia and Alzheimer's disease. Biochim. Biophys. Acta 1862, 887-900 (2016). [PubMed: 26705676]

55. Park L et al. Brain and Circulating Levels of A 1-40 Differentially Contribute to Vasomotor Dysfunction in the Mouse Brain. Stroke 44, 198-204 (2013). [PubMed: 23204056]

56. Poliakova T, Levin O, Arablinskiy A, Vasenina E \& Zerr I Cerebral microbleeds in early Alzheimer's disease. J. Neurol 263, 1961-1968 (2016). [PubMed: 27389080]

57. Manaenko A, Chen H, Zhang JH \& Tang J Comparison of Different Preclinical Models of Intracerebral Hemorrhage in Intracerebral Hemorrhage Research (eds. Zhang J \& Colohan A) 111, 9-14 (Springer Vienna, 2011).

58. Petraglia AL, Marky AH, Walker C, Thiyagarajan M \& Zlokovic BV Activated protein C is neuroprotective and mediates new blood vessel formation and neurogenesis after controlled cortical impact. Neurosurgery 66, 165-171; discussion 171-172 (2010). [PubMed: 20023547]

59. Toda N \& Okamura T Hyperhomocysteinemia impairs regional blood flow: involvements of endothelial and neuronal nitric oxide. Pflugers Arch. 468, 1517-1525 (2016). [PubMed: 27417104]

60. Sudduth TL, Powell DK, Smith CD, Greenstein A \& Wilcock DM Induction of hyperhomocysteinemia models vascular dementia by induction of cerebral microhemorrhages and neuroinflammation. J. Cereb. Blood Flow Metab. Off. J. Int. Soc. Cereb. Blood Flow Metab 33, 708-715 (2013). 
61. Hardigan T, Hernandez C, Ward R, Hoda MN \& Ergul A TLR2 knockout protects against diabetesmediated changes in cerebral perfusion and cognitive deficits. Am. J. Physiol. Regul. Integr. Comp. Physiol 312, R927-R937 (2017). [PubMed: 28336553]

62. Iordanova B, Li L, Clark RSB \& Manole MD Alterations in Cerebral Blood Flow after Resuscitation from Cardiac Arrest. Front. Pediatr 5, 174(2017). [PubMed: 28861407]

63. Khan MB et al. Chronic Remote Ischemic Conditioning Is Cerebroprotective and Induces Vascular Remodeling in a VCID Model. Transl. Stroke Res (2017). doi:10.1007/s12975-017-0555-1

64. Zhao Z, Nelson AR, Betsholtz C \& Zlokovic BV Establishment and Dysfunction of the BloodBrain Barrier. Cell 163, 1064-1078 (2015). [PubMed: 26590417]

65. Winkler EA et al. GLUT1 reductions exacerbate Alzheimer's disease vasculo-neuronal dysfunction and degeneration. Nat. Neurosci 18, 521-530 (2015). [PubMed: 25730668]

66. Guemez-Gamboa A et al. Inactivating mutations in MFSD2A, required for omega-3 fatty acid transport in brain, cause a lethal microcephaly syndrome. Nat. Genet 47, 809-813 (2015). [PubMed: 26005868]

67. Ben-Zvi A et al. Mfsd2a is critical for the formation and function of the blood-brain barrier. Nature 509, 507-511 (2014). [PubMed: 24828040]

68. Lacombe P, Oligo C, Domenga V, Tournier-Lasserve E \& Joutel A Impaired cerebral vasoreactivity in a transgenic mouse model of cerebral autosomal dominant arteriopathy with subcortical infarcts and leukoencephalopathy arteriopathy. Stroke 36, 1053-1058 (2005). [PubMed: 15817893]

69. Joutel A et al. Cerebrovascular dysfunction and microcirculation rarefaction precede white matter lesions in a mouse genetic model of cerebral ischemic small vessel disease. J. Clin. Invest 120, 433-445 (2010). [PubMed: 20071773]

70. Sharma A \& Shiras A Cancer stem cell-vascular endothelial cell interactions in glioblastoma. Biochem. Biophys. Res. Commun 473, 688-692 (2016). [PubMed: 26692486]

71. Dai M, Yang Y \& Shi X Lactate dilates cochlear capillaries via type V fibrocyte-vessel coupling signaled by nNOS. AJP Heart Circ. Physiol 301, H1248-H1254 (2011).

72. Martinez Sosa S \& Smith KJ Understanding a role for hypoxia in lesion formation and location in the deep and periventricular white matter in small vessel disease and multiple sclerosis. Clin. Sci. Lond. Engl 1979 131, 2503-2524 (2017).

73. Tang $\mathrm{P}$ et al. In vivo two-photon imaging of axonal dieback, blood flow, and calcium influx with methylprednisolone therapy after spinal cord injury. Sci. Rep 5, 9691(2015). [PubMed: 25989524]

74. Montagne A et al. Pericyte degeneration causes white matter dysfunction in the mouse central nervous system. Nat. Med (2018). doi:10.1038/nm.4482

75. Uhlirova $\mathrm{H}$ et al. Cell type specificity of neurovascular coupling in cerebral cortex. eLife 5, (2016).

76. Kasischke KA et al. Two-photon NADH imaging exposes boundaries of oxygen diffusion in cortical vascular supply regions. J. Cereb. Blood Flow Metab. Off. J. Int. Soc. Cereb. Blood Flow Metab 31, 68-81 (2011).

77. Sakadžić S et al. Large arteriolar component of oxygen delivery implies a safe margin of oxygen supply to cerebral tissue. Nat. Commun 5, 5734(2014). [PubMed: 25483924]

78. Shahram M \& Milanfar P Imaging below the diffraction limit: a statistical analysis. IEEE Trans. Image Process. Publ. IEEE Signal Process. Soc 13, 677-689 (2004).

79. Ram S, Ward ES \& Ober RJ Beyond Rayleigh's criterion: A resolution measure with application to single-molecule microscopy. Proc. Natl. Acad. Sci 103, 4457-4462 (2006). [PubMed: 16537357]

80. Rayleigh. XXXI. Investigations in optics, with special reference to the spectroscope. Philos. Mag. Ser 5 8, 261-274 (1879).

81. Nyquist H Certain Topics in Telegraph Transmission Theory. Trans. Am. Inst. Electr. Eng 47, 617644 (1928).

82. Shannon CE A Mathematical Theory of Communication. Bell Syst. Tech. J 27, 379-423 (1948).

83. Pawley JB Points, Pixels, and Gray Levels: Digitizing Image Data in Handbook Of Biological Confocal Microscopy (ed. Pawley JB) 59-79 (Springer US, 2006).

84. Hall CN et al. Capillary pericytes regulate cerebral blood flow in health and disease. Nature 508, 55-60 (2014). [PubMed: 24670647] 
85. Mishra A et al. Astrocytes mediate neurovascular signaling to capillary pericytes but not to arterioles. Nat. Neurosci 19, 1619-1627 (2016). [PubMed: 27775719]

86. Otsu $\mathrm{Y}$ et al. Calcium dynamics in astrocyte processes during neurovascular coupling. Nat. Neurosci 18, 210-218 (2015). [PubMed: 25531572]

87. Kornfield TE \& Newman EA Regulation of blood flow in the retinal trilaminar vascular network. J. Neurosci. Off. J. Soc. Neurosci 34, 11504-11513 (2014).

88. Biesecker KR et al. Glial Cell Calcium Signaling Mediates Capillary Regulation of Blood Flow in the Retina. J. Neurosci. Off. J. Soc. Neurosci 36, 9435-9445 (2016).

89. Drew PJ, Shih AY \& Kleinfeld D Fluctuating and sensory-induced vasodynamics in rodent cortex extend arteriole capacity. Proc. Natl. Acad. Sci. U. S. A 108, 8473-8478 (2011). [PubMed: 21536897]

90. Takano T et al. Astrocyte-mediated control of cerebral blood flow. Nat. Neurosci 9, 260-267 (2006). [PubMed: 16388306]

91. Hill RA et al. Regional Blood Flow in the Normal and Ischemic Brain Is Controlled by Arteriolar Smooth Muscle Cell Contractility and Not by Capillary Pericytes. Neuron 87, 95-110 (2015). [PubMed: 26119027]

92. Damisah EC, Hill RA, Tong L, Murray KN \& Grutzendler J A fluoro-Nissl dye identifies pericytes as distinct vascular mural cells during in vivo brain imaging. Nat. Neurosci (2017). doi: $10.1038 / \mathrm{nn} .4564$

93. Wei HS et al. Erythrocytes Are Oxygen-Sensing Regulators of the Cerebral Microcirculation. Neuron 91, 851-862 (2016). [PubMed: 27499087]

94. Chang C-I, Du Y, Wang J, Guo S-M \& Thouin PD Survey and comparative analysis of entropy and relative entropy thresholding techniques. IEE Proc. - Vis. Image Signal Process 153, 837(2006).

95. Drew PJ, Blinder P, Cauwenberghs G, Shih AY \& Kleinfeld D Rapid determination of particle velocity from space-time images using the Radon transform. J. Comput. Neurosci 29, 5-11 (2010). [PubMed: 19459038]

96. Summers PM, Taylor ZJ \& Shih AY Two-Photon Imaging of Cerebral Vasodynamics in Awake Mice During Health and Disease in Advances in Intravital Microscopy (ed. Weigert R) 25-43 (Springer Netherlands, 2014).

97. Art J Photon Detectors for Confocal Microscopy in Handbook Of Biological Confocal Microscopy (ed. Pawley JB) 251-264 (Springer US, 2006).

98. Baran U \& Wang RK Review of optical coherence tomography based angiography in neuroscience. Neurophotonics 3, 010902(2016). [PubMed: 26835484]

99. Srinivasan VJ et al. OCT methods for capillary velocimetry. Biomed. Opt. Express 3, 612-629 (2012). [PubMed: 22435106]

100. Ma Y et al. Wide-field optical mapping of neural activity and brain haemodynamics: considerations and novel approaches. Philos. Trans. R. Soc. Lond. B. Biol. Sci 371, (2016).

101. Wang $\mathrm{Y}$ et al. 3K3A-activated protein $\mathrm{C}$ stimulates postischemic neuronal repair by human neural stem cells in mice. Nat. Med 22, 1050-1055 (2016). [PubMed: 27548576]

102. Berndt N, Kann O \& Holzhütter H-G Physiology-Based Kinetic Modeling of Neuronal Energy Metabolism Unravels the Molecular Basis of NAD $(\mathrm{P}) \mathrm{H}$ Fluorescence Transients. J. Cereb. Blood Flow Metab 35, 1494-1506 (2015). [PubMed: 25899300]

103. Yaseen MA et al. In vivo imaging of cerebral energy metabolism with two-photon fluorescence lifetime microscopy of NADH. Biomed. Opt. Express 4, 307-321 (2013). [PubMed: 23412419]

104. Yaseen MA et al. Fluorescence lifetime microscopy of NADH distinguishes alterations in cerebral metabolism in vivo. Biomed. Opt. Express 8, 2368-2385 (2017). [PubMed: 28663879]

105. Baraghis E et al. Two-photon microscopy of cortical NADH fluorescence intensity changes: correcting contamination from the hemodynamic response. J. Biomed. Opt 16, 106003(2011). [PubMed: 22029350]

106. Zhao Y et al. In vivo monitoring of cellular energy metabolism using SoNar, a highly responsive sensor for NAD+/NADH redox state. Nat. Protoc 11, 1345-1359 (2016). [PubMed: 27362337] 
107. Mongeon R, Venkatachalam V \& Yellen G Cytosolic NADH-NAD(+) Redox Visualized in Brain Slices by Two-Photon Fluorescence Lifetime Biosensor Imaging. Antioxid. Redox Signal 25, 553-563 (2016). [PubMed: 26857245]

108. Vanderkooi JM, Maniara G, Green TJ \& Wilson DF An optical method for measurement of dioxygen concentration based upon quenching of phosphorescence. J. Biol. Chem 262, 54765482 (1987). [PubMed: 3571219]

109. Becker W Fluorescence lifetime imaging - techniques and applications: FLUORESCENCE LIFETIME IMAGING. J. Microsc 247, 119-136 (2012). [PubMed: 22621335]

110. Sakadzić $\mathrm{S}$ et al. Simultaneous imaging of cerebral partial pressure of oxygen and blood flow during functional activation and cortical spreading depression. Appl. Opt 48, D169-177 (2009). [PubMed: 19340106]

111. Wilson DF et al. Effect of hyperventilation on oxygenation of the brain cortex of newborn piglets. J. Appl. Physiol. Bethesda Md 1985 70, 2691-2696 (1991).

112. Wilson DF, Gomi S, Pastuszko A \& Greenberg JH Microvascular damage in the cortex of cat brain from middle cerebral artery occlusion and reperfusion. J. Appl. Physiol. Bethesda Md 1985 74, 580-589 (1993).

113. Yaseen MA et al. Optical monitoring of oxygen tension in cortical microvessels with confocal microscopy. Opt. Express 17, 22341-22350 (2009). [PubMed: 20052157]

114. Sakadzić S et al. Two-photon high-resolution measurement of partial pressure of oxygen in cerebral vasculature and tissue. Nat. Methods 7, 755-759 (2010). [PubMed: 20693997]

115. Lecoq J et al. Simultaneous two-photon imaging of oxygen and blood flow in deep cerebral vessels. Nat. Med 17, 893-898 (2011). [PubMed: 21642977]

116. Devor A et al. 'Overshoot' of $\mathrm{O}_{2}$ is required to maintain baseline tissue oxygenation at locations distal to blood vessels. J. Neurosci. Off. J. Soc. Neurosci 31, 13676-13681 (2011).

117. Lyons DG, Parpaleix A, Roche M \& Charpak S Mapping oxygen concentration in the awake mouse brain. eLife 5, e12024(2016). [PubMed: 26836304]

118. Kazmi SMS et al. Three-dimensional mapping of oxygen tension in cortical arterioles before and after occlusion. Biomed. Opt. Express 4, 1061(2013). [PubMed: 23847732]

119. Spencer JA et al. Direct measurement of local oxygen concentration in the bone marrow of live animals. Nature 508, 269-273 (2014). [PubMed: 24590072]

120. Yaseen MA et al. Multimodal optical imaging system for in vivo investigation of cerebral oxygen delivery and energy metabolism. Biomed. Opt. Express 6, 4994-5007 (2015). [PubMed: 26713212]

121. Kalmbach AS \& Waters J Brain surface temperature under a craniotomy. J. Neurophysiol 108, 3138-3146 (2012). [PubMed: 22972953]

122. Shirey MJ et al. Brief anesthesia, but not voluntary locomotion, significantly alters cortical temperature. J. Neurophysiol 114, 309-322 (2015). [PubMed: 25972579]

123. Goldey GJ et al. Removable cranial windows for long-term imaging in awake mice. Nat. Protoc 9 , 2515-2538 (2014). [PubMed: 25275789]

124. Rumsey WL, Vanderkooi JM \& Wilson DF Imaging of phosphorescence: a novel method for measuring oxygen distribution in perfused tissue. Science 241, 1649-1651 (1988). [PubMed: 3420417]

125. Mik EG, van Leeuwen TG, Raat NJ \& Ince C Quantitative determination of localized tissue oxygen concentration in vivo by two-photon excitation phosphorescence lifetime measurements. J. Appl. Physiol. Bethesda Md 1985 97, 1962-1969 (2004).

126. Holtmaat A et al. Long-term, high-resolution imaging in the mouse neocortex through a chronic cranial window. Nat. Protoc 4, 1128-1144 (2009). [PubMed: 19617885]

127. Polesskaya O et al. Detection of Microregional Hypoxia in Mouse Cerebral Cortex by Twophoton Imaging of Endogenous NADH Fluorescence. J. Vis. Exp (2012). doi:10.3791/3466

128. Shih AY, Mateo C, Drew PJ, Tsai PS \& Kleinfeld D A polished and reinforced thinned-skull window for long-term imaging of the mouse brain. J. Vis. Exp. JoVE (2012). doi:10.3791/3742

129. Tallquist MD, French WJ \& Soriano P Additive effects of PDGF receptor beta signaling pathways in vascular smooth muscle cell development. PLoS Biol. 1, E52(2003). [PubMed: 14624252] 
130. Schneider CA, Rasband WS \& Eliceiri KW NIH Image to ImageJ: 25 years of image analysis. Nat. Methods 9, 671-675 (2012). [PubMed: 22930834]

131. Schindelin J et al. Fiji: an open-source platform for biological-image analysis. Nat. Methods 9 , 676-682 (2012). [PubMed: 22743772]

132. Sakadžić S et al. Cerebral blood oxygenation measurement based on oxygen-dependent quenching of phosphorescence. J. Vis. Exp. JoVE (2011). doi:10.3791/1694

133. Sinks LE et al. Two-photon microscopy of oxygen: polymersomes as probe carrier vehicles. J. Phys. Chem. B 114, 14373-14382 (2010). [PubMed: 20462225] 


\section{Box 2 Two-photon set up for diameter, velocity, and NADH imaging TIMING:}

\section{0 min}

1. Place heating pad, pulse oximeter, and isoflurane apparatus in convenient locations around the microscope. Turn on the heating pad and allow it to warm up for 10 minutes. If the isoflurane vaporizor uses a heating element for vaporization, turn on and allow it to warm up for $10 \mathrm{~min}$.

2. Start two-photon microscope and laser, and configure for imaging by first seting up widefield fluorescence imaging for positioning. This will be used to locate the region of interest. Set the green emission filter to acquire FITC emission (approx. 500-550 nm) while using a blue excitation light (approximately 470-500 nm). Even in the absence of exogeneous contrast agents, blue excitation will generate sufficient tissue autofluorescence to make a diffuse green glow from the tissue and dark shadows where blood vessels are present.

3. Set two-photon excitation wavelength and emission filter depending on the fluorophore used. See Protocols steps 18B.ii and 18C.iv for specific excitation and emission conditions for diameter and velocity, or NADH imaging, respectively.

4. Set the microscope to output a trigger signal while imaging and/or producing linescans.

5. Set stimulus source to deliver electrical current stimulus via needle electrode probes. Initially set the amplitude to $\sim 1 \mathrm{~mA}$. We used $2 \mathrm{~ms}$ pulses at a rate of $10 \mathrm{~Hz}$ for $10 \mathrm{~s}$, triggered by the DigiData.

6. Set up the DigiData to trigger the electrical stimulus and simultaneously record the microscope output trigger. This information will be used later to register the stimulus timing to the image or linescan acquisition. There should be a 10-20 s delay between DigiData recording start and stimulus trigger start, so that a baseline series of images/linescans can be acquired. The imaging should be continued for $60 \mathrm{~s}$ after the stimulus ends. When running the experiment, the DigiData is started first, and image acquisition started manually immediately after.

CAUTION: Direct eye exposure to the laser light used in our experiments can lead to permanent eye damage. Please carefully follow all safety procedures. 


\section{Box 3 IOS setup TIMING: 1-2 hr for initial equipment and software setup, $\sim 10$ min subsequently}

1. Position dissecting scope on table. Install high speed cMOS camera and filter in filter holder or in space between scope and camera (see Figure 6 inset).

2. Mount LED array to table and angle to illuminate the area below the dissecting scope (Figure 6). Connect leads to power supply.

3. Prepare camera and software. In camera software (BV_Ana for the MiCAM02 camera) set camera to $30 \mathrm{~ms} /$ frame $(33 \mathrm{~Hz})$. For the MiCAM02 camera, pixels were set to $2 \mathrm{x}$ binning. Set trigger pulse to start at desired time during image sequence, and for desired duration. Ensure that stimulus trigger input is attached to the camera output trigger.

4. Set up stimulus. Follow option A for vibration stimulus or option B for electrical stimulus. In our hands, vibration stimulus is used for short stimulus duration protocols, and electrical stimulus for long stimulus duration protocols or to match stimulus protocols used in other experiments such as measurement of vessel diameter and velocity (step 18B), or NADH (step 18C).

A. Vibration stimulus set-up:

i. Connect or solder vibration motor in series with resistor (one lead of motor to one lead of resistor), then solder the remaining two leads to the positive and negative wires or cable leading to the BNC connector. Test that electrical contact has been made between all parts. A multimeter should read a non-infinite resistance between the positive and negative wires. Use electrical tape to wrap the exposed wires and provide additional support.

ii. Attach the wires/cable leading to the motor to the positioning support. This will keep the motor off the table and reduce vibration artifacts when imaging. Attach the BNC connector to the stimulus output trigger of the camera.

iii. Test that the camera trigger signal generates a vibration. Hold motor between fingers and trigger the stimulus. A vibration should be felt. To test a setup use a stimulus duration between $100 \mathrm{~ms}$ and $10 \mathrm{~s}$. Test before every experiment.

?Troubleshooting

B. Electrical stimulus set-up: 
i. Set stimulator to appropriate stimulus. We start with $\sim 1 \mathrm{~mA}$ amplitude, $2 \mathrm{~ms}$ pulses at a rate of $10 \mathrm{~Hz}$. Connect to the camera trigger signal output.

ii. Connect leads of needle electrodes to stimulator.

5. Turn on LEDs and allow them to stabilize for about 5 min before running experiment.

CAUTION: Direct eye exposure to the LED light used in our experiments can lead to permanent eye damage. Please carefully follow all safety procedures. 


\section{Box 4 Two-photon set up for absolute tissue $\mathrm{pO}_{2}$ measurements TIMING: 30}

\section{$\min$}

1. Start microscope software and LabView programs for controlling phosphorescent dye data acquisition. Control of the galvanometer scanners, electro-optic modulator (EOM; used to produce excitation gates), and all other equipment is performed by a custom program written in LabView 114,132 . During the measurements, the software changes the position of the laser beam focus by adjusting the position of the galvanometer scanning mirrors, controls the excitation beam gating by EOM, and acquisition of the signals.

2. The microscope's EOM extinction ratio should be maximized by following the alignment instructions provided by the manufacturer. In our setup, successful measurements of the PtP-C343 phosphorescence lifetimes are obtained with an EOM extinction ratio greater than 400. If other optical elements are used to gate the laser excitation instead of EOM (e.g., acoustooptical deflector), they should exhibit at least similarly large extinction ratio.

3. Set two-photon excitation to a wavelength and emission filter suitable for the dye used. See Protocols section "Two-photon imaging of absolute $\mathrm{pO}_{2}$ " for specific excitation and emission conditions.

4. For imaging, carefully enclose the microscope to prevent exposure of the detector to any sources of light from the room with wavelengths that can pass through the emission filter. In the absence of the ideal microscope enclosure, all sources of light in the room should be turned off, covered, or significantly dimmed during the experiment. 
Box 5. Mouse setup for two-photon diameter, RBC velocity, or NADH imaging

TIMING: 10-20 min

1. Perform IOS measurement to identify the brain region responsive to stimulus (activation center) by following IOS imaging procedure (steps 18A.i-xii).

2. Position mouse and stereotaxic frame, and heating pad to maintain mouse core body temperature, on the microscope.

3. Position $\mathrm{SpO}_{2}$ probe on one of the mouse feet

CAUTION: Ensure proper depth of anesthesia before continuing. Mouse should not respond to tow pinch.

4. Insert electrical probes into the mouse contralateral hind limb and adjust stimulus, as for IOS imaging (step 18A.iii)

5. Locate the surface of the brain using widefield fluorescence imaging through the microscope eyepiece. With the blue light excitation and green fluorescence emission (FITC channel), it is possible to identify the brain tissue surface and the pial vasculature. 


\section{Box 1 Two-photon imaging of absolute $\mathrm{pO}_{2}$}

TIMING: Absolute $\mathrm{pO}_{2}$ measurements take up to $4 \mathrm{hr}$. This includes $\sim 2$-hr-long animal preparation (e.g., surgical procedures, dye injection) and $~ 2$-hr-long measurement of tissue $\mathrm{pO}_{2}$ with the two-photon microscope. The image analysis takes $2 \mathrm{~h}$ per mouse.

1. Anesthetize the mouse with isoflurane. Maintain the mouse under 1-1.5\% isoflurane in a mixture of air and oxygen. Maintain mouse body temperature at $37 \pm 0.1^{\circ} \mathrm{C}$ with a homeothermic heating pad.

2. Perform tracheotomy. Ventilate mouse with the same mixture of $1-1.5 \%$ isoflurane in a mixture of air and oxygen. Connect equipment to monitor end tidal $\mathrm{pCO}_{2}$.

3. Catheterize the femoral artery. Connect equipment to monitor the heart rate, blood pressure, and to sample systemic blood gases $\left(\mathrm{pCO}_{2}\right.$ and $\left.\mathrm{pO}_{2}\right)$ and blood $\mathrm{pH}$.

CRITICAL STEP: During mouse preparation procedure and experiment, adjust ventilation and anesthesia to keep the blood pressure and blood gases within the normal physiological range. We maintain arterial $\mathrm{pO}_{2}$ at $115 \pm 15 \mathrm{mmHg}$, expired end tidal $\mathrm{pCO}_{2}$ at $37 \pm 2 \mathrm{mmHg}$, mean arterial blood pressure at $100 \pm 10 \mathrm{mmHg}$, and blood $\mathrm{pH}$ at $7.37 \pm 0.02$.

4. Perform cranial window installation as described in steps $1-15$, leaving the window open. Remove the dura (step 14).

5. Following the supplementary method in Goldey, et al ${ }^{123}$, insert a glass micropipette loaded with the PtP-C343 dye solution about $400 \mu \mathrm{m}$ deep into the cortex. Slowly inject about $0.1 \mu \mathrm{L}$ of dye into the brain. Successful injection of PtP-C343 typically creates a very light yellow circle up to $\sim 1 \mathrm{~mm}$ in diameter centered at the injection site.

CRITICAL STEP: Injection of the dye should be performed at a location away from large pial arterioles and veins, to minimize the chance of puncturing a larger vessel during micropipette insertion into the brain. An injection site close to the edge of the cranial window may result in limited imaging depth due to partial obstruction of the excitation and emission light by the window edges.

6. Seal cranial window with a glass coverslip and cyanoacrilate gel glue following steps 16-17.

CRITICAL STEP: Care should be taken to avoid brain edema formation and any blood leakage over the cortical surface during the cranial window installation procedure.

7. For imaging, discontinue isoflurane anesthesia and replace with alphachloralose anesthesia via IV $(50 \mathrm{mg} / \mathrm{kg} / \mathrm{h})$. Deliver Alpha-chloralose via the femoral artery line every $\sim 30 \mathrm{~min}$. 
CRITICAL STEP: Volume and timing of anesthesia injections should be adjusted based on continuous monitoring of the mean arterial blood pressure, heart rate, and end tidal $\mathrm{pCO} 2$.

8. Position the mouse under the two-photon microscope modified for $\mathrm{pO}_{2}$ imaging.

9. Center the cranial window in the field of view using the low-magnification (4x) objective. The animal head may need to be tilted such that the pial surface inside the cranial window is parallel to the focal plane.

CRITICAL STEP: The angle between the brain surface and the objective imaging plane should be minimal. Otherwise, it may impair the quality of the data due to significant changes in the imaging depth over the field of view.

10. Inject $20 \mu \mathrm{L}$ of Fluorescein isothiocyanate-Dextran (2000 kDa) dye solution via the femoral artery line.

CAUTION: Keep the injected volume of fluorescein dye to a minimum to avoid affecting animal physiology.

11. Acquire "navigation images" with the $4 x$ objective to use as maps for subsequent imaging. Acquire an image of the pial vasculature inside the cranial window with the digital microscope (CCD) camera under green light illumination (Figure 8a). Tune the two-photon laser excitation to a wavelength between $800-820 \mathrm{~nm}$, and set filters to collect fluorescence emission in green, $500-550 \mathrm{~nm}$. Acquire a quick two-photon image stack of the microvasculature over the same field of view.

12. Switch to the $20 \mathrm{x}$ water immersion objective and affix the objective heater. Set objective heater temperature to achieve $37^{\circ} \mathrm{C}$ temperature of water between objective lens and cranial window. While the temperature is stabilizing, look over navigation images to start identifying potential imaging locations.

13. Acquire two-photon images of fluorescein dye-labeled blood plasma to identify the desired imaging location(s) and depth(s) (Figure 8b).

14. Tune the laser to $920 \mathrm{~nm}$ excitation and set to collect phosphorescence emission: $10 \mu$ s-long excitation gates, followed by a 300-500 $\mu$ s-long phosphorescence collection period. Collect phosphorescence emission at wavelengths between $650-800 \mathrm{~nm}$. Acquire a phosphorescence survey scan, i.e. a raster scan of phosphorescence intensity, in order to gauge the strength of the phosphorescence signal at the image location and depth chosen (Figure $8 c)$.

CAUTION: Use the minimal laser power possible for phosphorescence excitation to avoid excitation saturation of the probe near the focal volume. Saturation would expand the effective focal volume and essentially lower spatial resolution ${ }^{133}$. 
15. Use the LabView program to set up phosphorescent dye measurement points in a grid pattern across the chosen imaging location (Figure 8c). Use the software to excite dye and record phosphorescence decays at each grid point. Use 500 - 2000 excitation/collection cycles at each grid point (e.g. Figure $8 d)$.

?Troubleshooting

CRITICAL STEP: In our experience, successful PtP-C343 lifetime measurement requires greater than 10-fold SNR between the photon count rate at the beginning of the phosphorescence decay and the residual background signal at the end of the decay.

16. Repeat steps Box 111-15 for any other imaging depth(s) and location(s).

17. Inject $0.1 \mathrm{~mL}$ of Rhodamine $\mathrm{B}$ dye solution into the vasculature via the femoral artery to robustly label vasculature. Tune the two-photon laser to $800-850 \mathrm{~nm}$ to excite Rhodamine B fluorescence. Set the emission filter to collect Rhodamine B fluorescence ( $\sim 610 \mathrm{~nm}$ peak emission wavelength).

18. Acquire a microvascular stack image of rhodamine $B$ fluorescence through the region(s) imaged with the PtP-C343 phosphorescent dye with standard two-photon imaging (Figures 8g,h).

19. Once data acquisition is complete, mouse can either be sacrificed in accordance with Institutional and National regulations, or allowed to recover and returned to its home cage.

CAUTION Ensure that experiments involving live animals conform to Institutional and National regulations.

PAUSE POINT: Absolute $\mathrm{pO}_{2}$ image analysis can be performed at a later day or time, after the imaging experiments are complete.

20. Absolute $\mathrm{pO} 2$ image processing is performed with custom routines in Matlab ${ }^{114}$. For each grid point, fit a single exponential function to the phosphorescence decay using a nonlinear least squares algorithm. Record the phosphorescence lifetime.

21. Using the Stern-Volmer equation and calibration parameters provided with the dye ${ }^{48,114}$, convert the phosphorescence lifetimes to $\mathrm{pO}_{2}$ values.

22. The $\mathrm{pO}_{2}$ values can be plotted relative to the vascular locations. Align the Rhodamine B vascular images to the fluorescein images. The Rhodamine B images will have a more robust signal than the fluorescein vascular images. Figure 8e illustrates color coded $\mathrm{pO}_{2}$ values overlaid on a vascular projection, for example. 


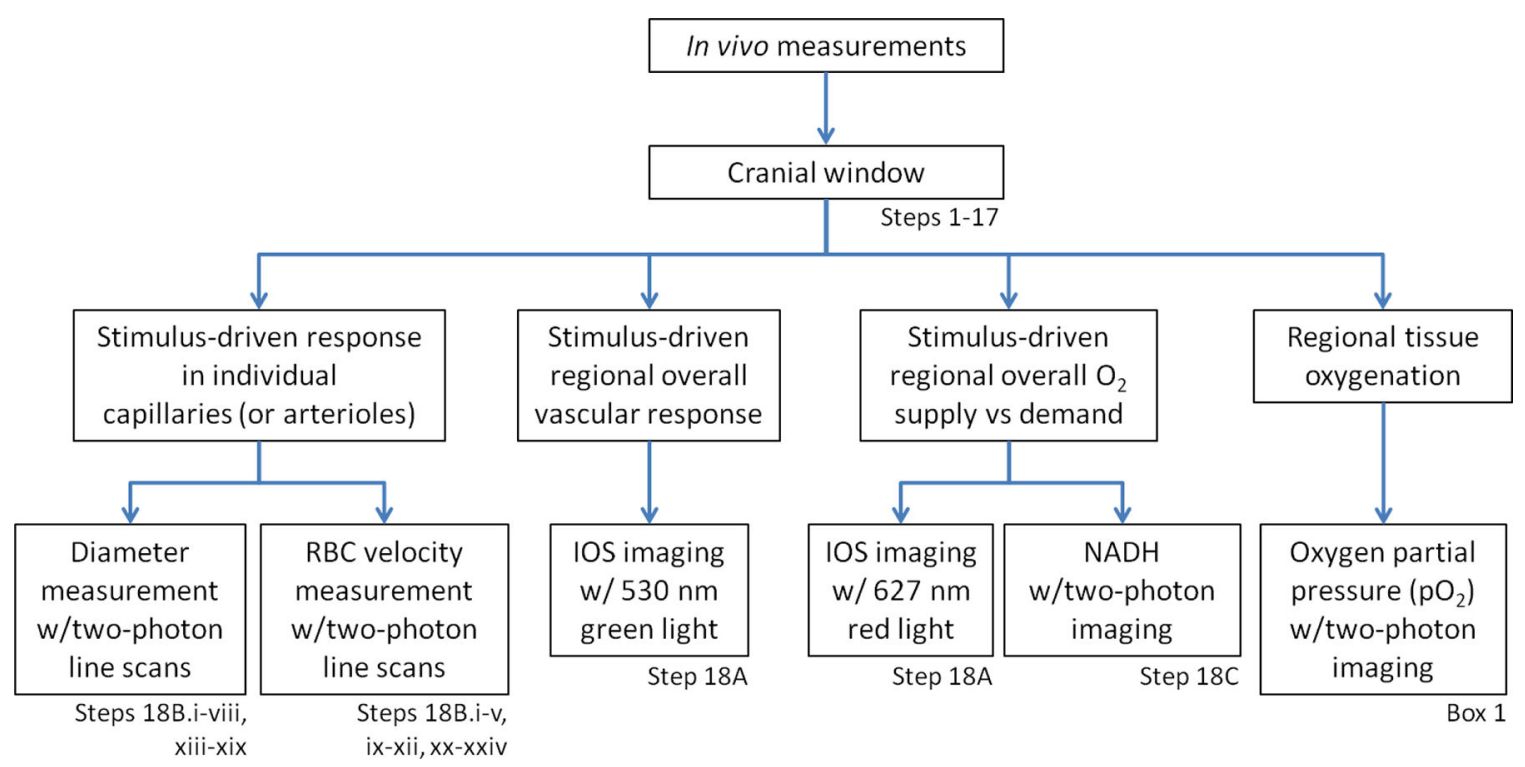

Figure 1: A workflow describing different in vivo methods to study brain hemodynamic responses and tissue oxygenation in mice.

RBC: red blood cell, IOS: intrinsic optical signal, NADH: nicotinamide adenine dinucleotide, $\mathrm{O}_{2}$ : oxygen. 
a

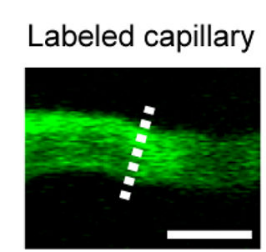

Dashed line:

Example linescan location b

kymograph
C

capillary diameter vs. Time

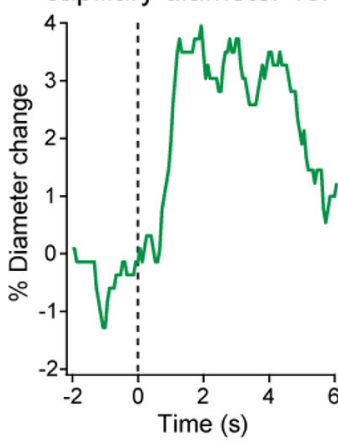

Obtain linescan (kymograph)

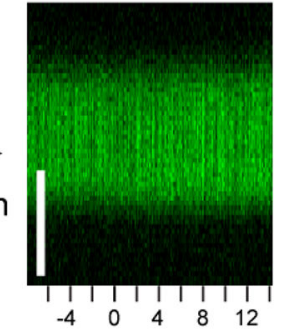

Time (s)

Measure diameter

1. Gaussian filter

2. Threshold

3. Obtain capillary diameter

4. Normalize to basal diameter

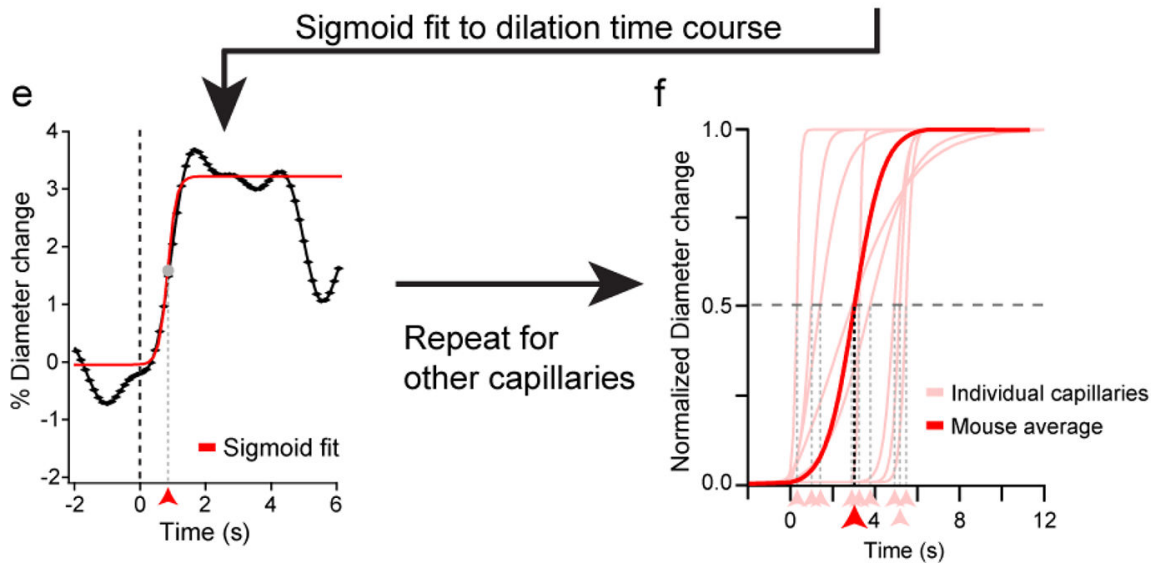

Figure 2: Two-photon microscopy measurements of individual brain capillary diameter changes in somatosensory cortex in response to electrical stimulus in an anesthetized wild type mouse. (Step 18B) (a) An individual FITC-dextran (70 kDa) dye-labeled capillary is shown with a linescan location perpendicular to the capillary marked for diameter measurement (scale bar is $5 \mu \mathrm{m}$ ). (b) High resolution linescans perpendicular to the capillary are acquired over time before, and in response to an electrical hind limb stimulus starting at $0 \mathrm{~s}(10 \mathrm{~s}$ duration at 10 $\mathrm{Hz}, 2 \mathrm{~ms}$ pulses) using a two-photon microscope. The linescans are built up over time into a linescan image or kymograph (scale bar is $2.5 \mu \mathrm{m}$ ). (c) The kymograph image then undergoes pre-threshold filtering with a 2D Gaussian filter. Then the image is thresholded to separate the vascular signal from the background (see steps 18B.xiii-xiv), yielding a binary black and white image from which the capillary diameter is determined for each line to create a time course trace of capillary diameter changes in response to stimulus. The 
diameter time course trace is normalized to its basal value (see step 18B.xvii), and diameter changes are expressed as a percentage of basal diameter (set to 0), resulting in the "raw" capillary diameter time course trace. A dashed line denotes the stimulus start. (d) The raw time course data is subsequently subjected to low-pass, notch, and box filters to reduce noise and breathing artifacts, resulting in the final "Filtered" capillary diameter time course trace in response to stimulus, as a percentage of basal diameter (see steps 18B.xvii). (e) To determine the time to reach $50 \%$ peak dilation, a sigmoid curve is fit to the diameter dilation time course. The time at which the sigmoid curve reaches 50\% maximal value corresponds to the capillary time to 50\% peak dilation, and is marked with a gray dot and dropline to the time axis. Arrowhead indicates time to 50\% peak dilation for this vessel. (f) The measurement process and sigmoid fit is repeated for all diameter time course traces derived from individual capillary responses to stimulus recorded in a single animal. Sigmoid fits were normalized between basal diameter (set to 0 ) and maximal diameter change in response to stimulus (set to 1). The average sigmoid curve fit (thick red line) were derived from the individual sigmoid fits from multiple capillaries from the same wild type mouse. A horizontal dashed line at 0.5 indicates $50 \%$ of maximal value, and arrowheads below the time axis indicate times when individual and mouse average capillary sigmoid fits reach $50 \%$ maximal diameter. The same process can be used to measure diameter changes in response to stimulus in arteriole vessels. This procedure was approved by the Institutional Animal Care and Use Committee at the University of Southern California with National Institutes of Health guidelines. 
a

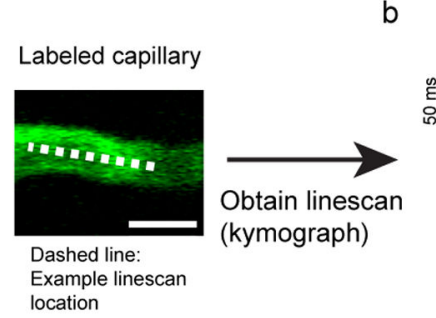

C

Raw capillary
RBC velocity vs. Time

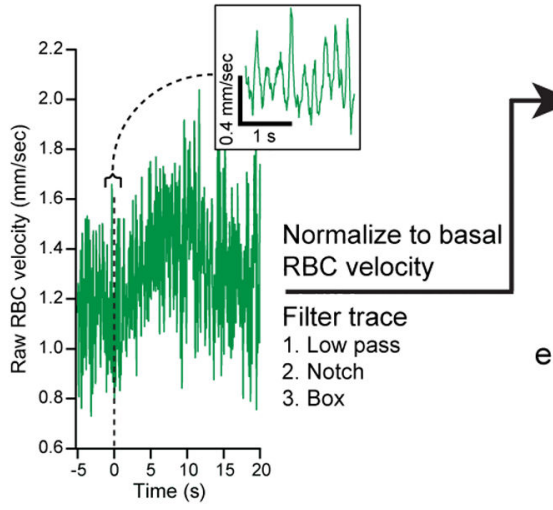

Repeat for other capillaries

$f$

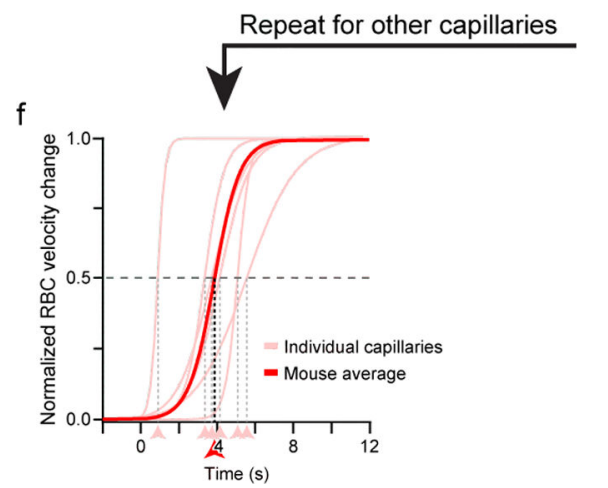

d

e
RBC

kymograph kymograph
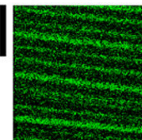

7

Measure RBC velocity

Matlab routine

Filtered capillary RBC velocity vs. Time


Figure 3: Two-photon microscopy measurements of vascular RBC velocity changes in response to electrical stimulus in somatosensory cortex of an anesthetized wild type mouse.

(Step 18B) (a) An individual FITC-dextran (70 kDa) dye-labeled capillary is shown with a linescan location along the center of the capillary marked for RBC velocity measurement (scale bar is $5 \mu \mathrm{m}$ ). (b) High speed linescans of the capillary are acquired using a twophoton microscope. The linescans are built up over time into a linescan image or RBC kymograph (scale bar is $5 \mu \mathrm{m}$ ). Dark stripes indicate the passage of RBCs through the vessel. The angle of the stripes indicate the speed at which the RBCs are traveling, and are used to measure RBC velocity. (c) Velocity information is extracted from the kymograph using a Matlab routine developed by Kim, et $\mathrm{al}^{41}$ in response to an electrical hind limb stimulus starting at $0 \mathrm{~s}(10 \mathrm{~s}$ duration at $10 \mathrm{~Hz}, 2 \mathrm{~ms}$ pulses $)$. A dashed line denotes the stimulus start. The resulting "Raw" capillary RBC velocity time course trace typically exhibits a strong heartbeat artifact. Inset: Enlarged view of a portion of velocity trace indicated by the bracket illustrating a heartbeat artifact of similar magnitude as reported 
by ${ }^{41,93}$. (d) The raw capillary RBC velocity trace is subsequently normalized to its baseline velocity, and $\mathrm{RBC}$ velocity changes are expressed as a percentage of basal baseline velocity (set to 0 ). The RBC velocity time course traces is passed through low-pass, notch, and box filters to reduce noise, heartbeat, and breathing artifacts, resulting in the final "Filtered" capillary RBC velocity trace (see text for details). (e) To determine the time to reach 50\% peak velocity, a sigmoid curve is fit to the RBC velocity increase time course, similar to diameter measurements. The capillary time to $50 \%$ peak velocity corresponds to the sigmoid $50 \%$ peak point, and is marked with a gray dot and dropline to the time axis. Arrowhead indicates time to $50 \%$ peak RBC velocity for this capillary. (f) The measurement process and sigmoid fit is repeated for all capillary RBC velocity data recorded in an individual animal. Sigmoid fits were normalized between basal RBC velocity (set to 0 ) and maximal velocity change in response to stimulus (set to 1). The average sigmoid curve fit (thick red line) was derived from the individual sigmoid fits from multiple capillaries from the same wild type mouse. A horizontal dashed line at 0.5 indicates $50 \%$ of maximal value, and arrowheads below the time axis indicate time individual and average capillary sigmoid fits reach 50\% maximal velocity. The same process is used to measure $\mathrm{RBC}$ velocity changes in response to stimulus in arteriole vessels. This procedure was approved by the Institutional Animal Care and Use Committee at the University of Southern California with National Institutes of Health guidelines. 
a

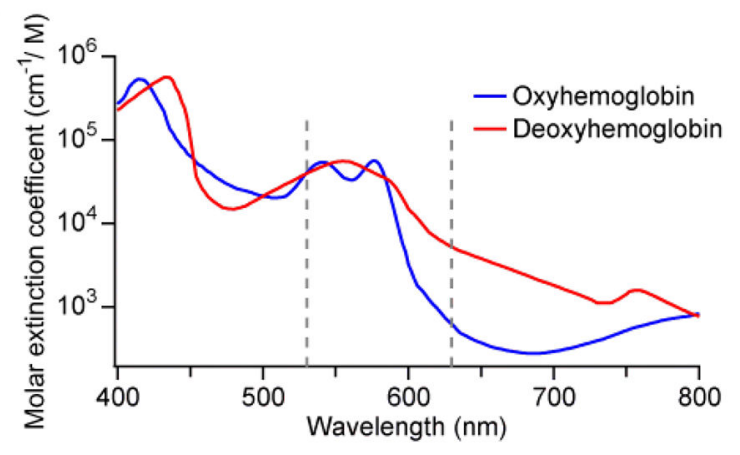

b

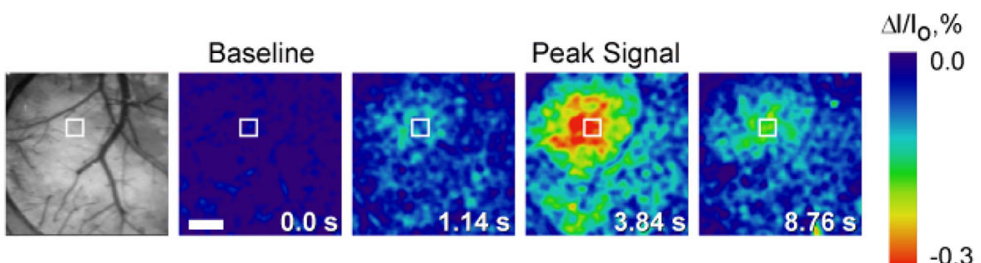

C

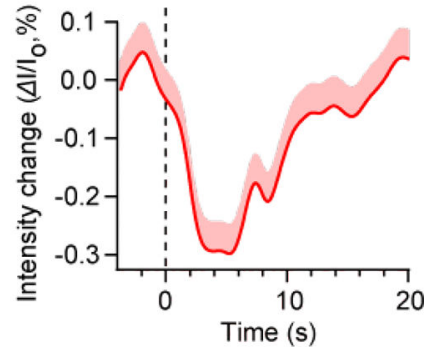

Figure 4: IOS imaging of regional hemodynamic response with green $530 \mathrm{~nm}$ illumination in an anesthetized mouse in somatosensory cortex.

(Step 18A) (a) The absorption spectra of oxy- and deoxyhemoglobin, expressed as the molar extinction coefficient. Larger values indicate greater absorption. Dashed lines indicate wavelengths used for green $(530 \mathrm{~nm})$ and red $(630 \mathrm{~nm})$ IOS imaging. (b) Image of the surface of the somatosensory cortex through a cranial window during IOS imaging (far left), and pseudocolored IOS signal changes in the same area in response to hind limb electrical stimulus starting at $0 \mathrm{~s}(10 \mathrm{~s}$ duration at $10 \mathrm{~Hz}, 2 \mathrm{~ms}$ pulses). The IOS image data set is an average from 10 individual stimulus trials. IOS Images were normalized to their average prestimulus baseline values (set to 0 ) for each pixel, and expressed as percent change in reflected intensity (I) from baseline $\left(\Delta \mathrm{I} / \mathrm{I}_{0}, \%\right)$. Images were smoothed with a $3 \mathrm{D}(\mathrm{x}, \mathrm{y}$, time) spatial filter. Boxes indicate region of interest (ROI) for panel $b$, placed to avoid any large surface vessels. Scale bar is $0.5 \mathrm{~mm}$. (c) Green IOS time course of the parenchymal ROI in (b) in response to stimulus. Curve is an average of 10 trials, and shaded area indicates standard deviation. A dashed line denotes the stimulus start. Green IOS signals are typically monophasic in vivo with a negative deflection indicating increased hemoglobin absorbance due to increase in blood flow. This procedure was approved by the Institutional Animal Care and Use Committee at the University of Southern California with National Institutes of Health guidelines. Panel a data compiled by Dr. Scott Prahl, Oregon Institute of Technology (http://omlc.org/spectra/hemoglobin/index.html). 
a

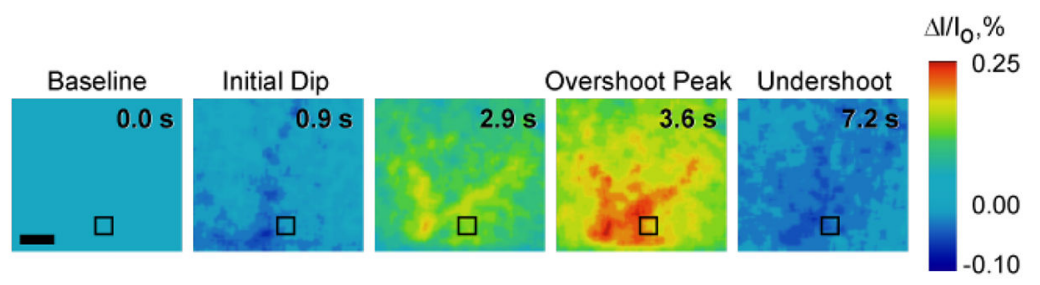

b

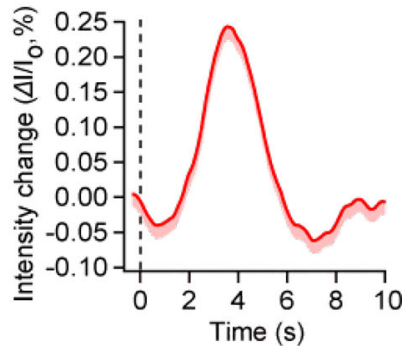

Figure 5: IOS imaging of regional hemodynamic response with red 630nm illumination in an anesthetized mouse in somatosensory cortex.

(Steps 18A) (a) Pseudocolored red IOS signal changes in response to hind limb mechanical vibration stimulus starting at $0 \mathrm{~s}$ (300 ms duration). The IOS image data set is an average from 10 individual stimulus trials. IOS Images were normalized to their average prestimulus basal values (set to 0 ) for each pixel, and expressed as percent change in reflected intensity (I) from baseline $\left(\Delta \mathrm{I} / \mathrm{I}_{\mathrm{O}}, \%\right)$. Images were smoothed with a $3 \mathrm{D}$ filter. Boxes indicate region of interest (ROI) for panel $\mathbf{b}$, placed to avoid any large surface vessels. Scale bar is $0.5 \mathrm{~mm}$. (b) Red IOS time course of the parenchymal ROI in (a) in response to stimulus. Curve is an average of 10 trials, and shaded area indicates standard deviation. A dashed line denotes the stimulus start. Red IOS signals are typically multiphasic in vivo with a negative "initial dip," followed by a positive "overshoot" and second dip or "undershoot." The overshoot indicates deoxyhemoglobin washout and is a measure of oxygen delivery to the active brain region. This procedure was approved by the Institutional Animal Care and Use Committee at the University of Southern California with National Institutes of Health guidelines. 


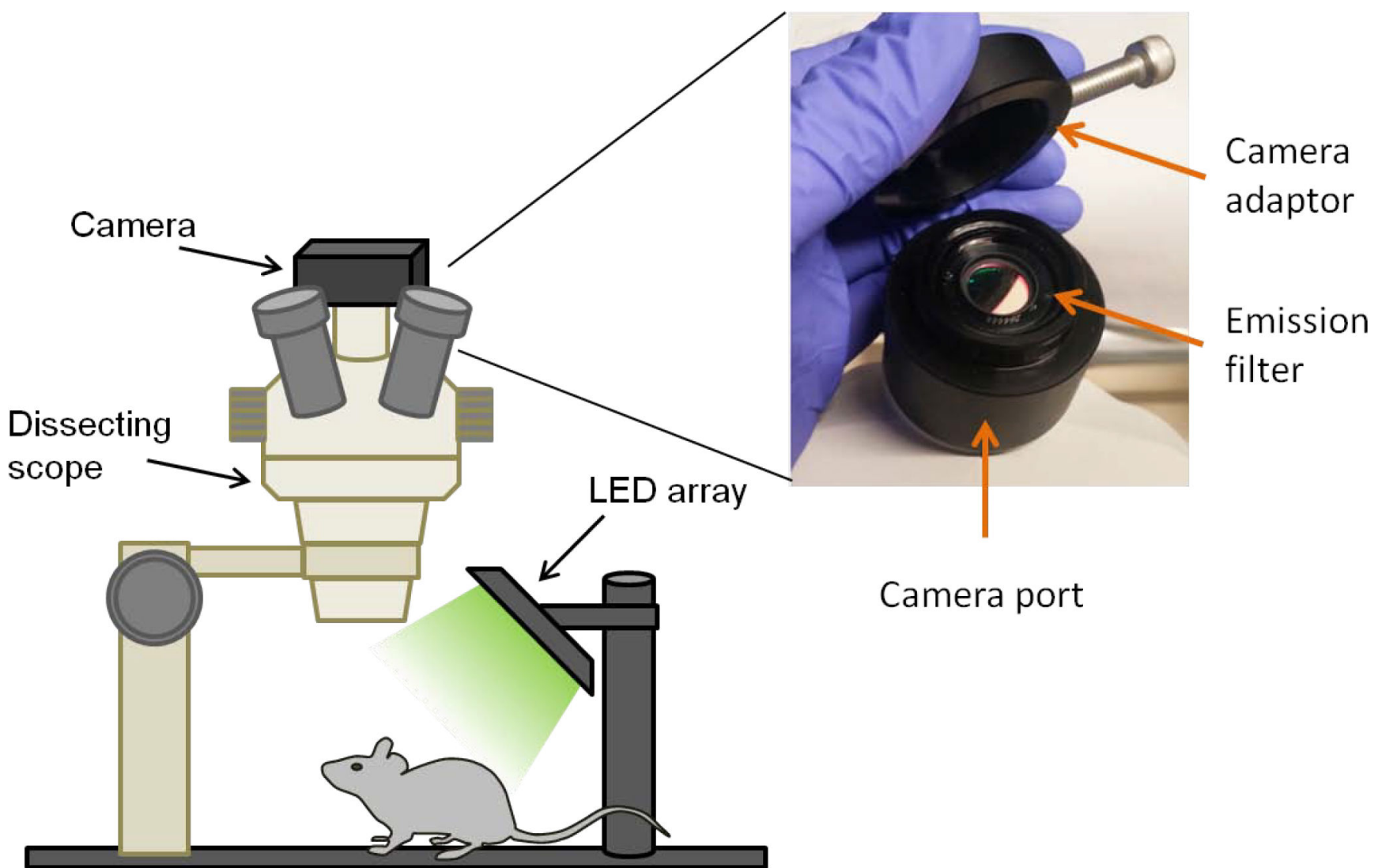

\section{Anesthetized mouse on steriotaxic frame (not shown)}

Figure 6: Dissecting scope configured for IOS imaging.

A simplified IOS imaging set up was configured from a dissecting scope with a camera port, a CMOS camera, and a $2 \times 3$ LED array angled to illuminate the imaging area (Box 3). An anesthetized mouse with a cranial window sits in a steriotaxic frame below the scope for imaging. The light reflected by the brain surface is collected through the scope by the camera after passing through an emission filter to select the reflected light from the LEDs. Inset: In dissecting scopes without a dedicated filter holder, a 15-18 mm diameter emission filter is placed in the space between the camera port (tube) lens, and the camera adaptor. 
a
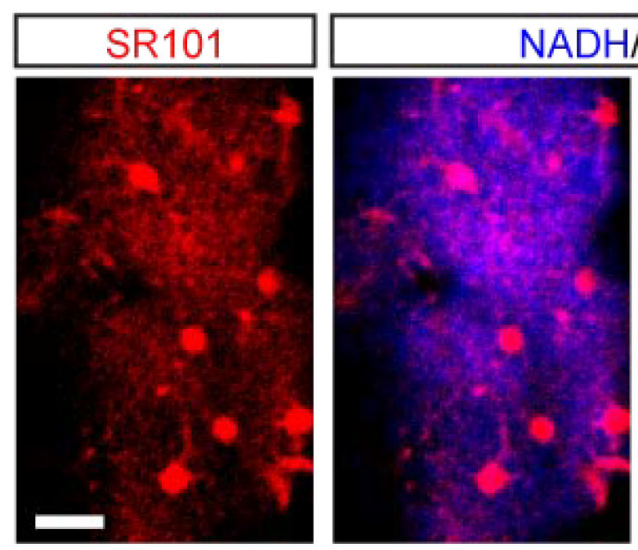

Baseline

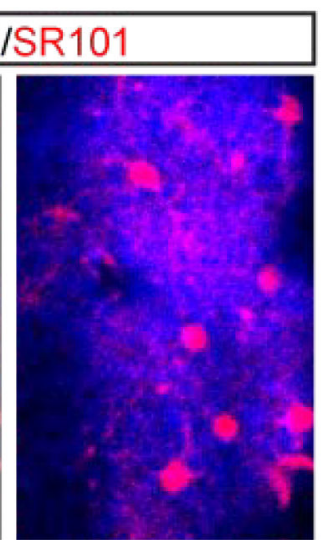

Peak NADH change b

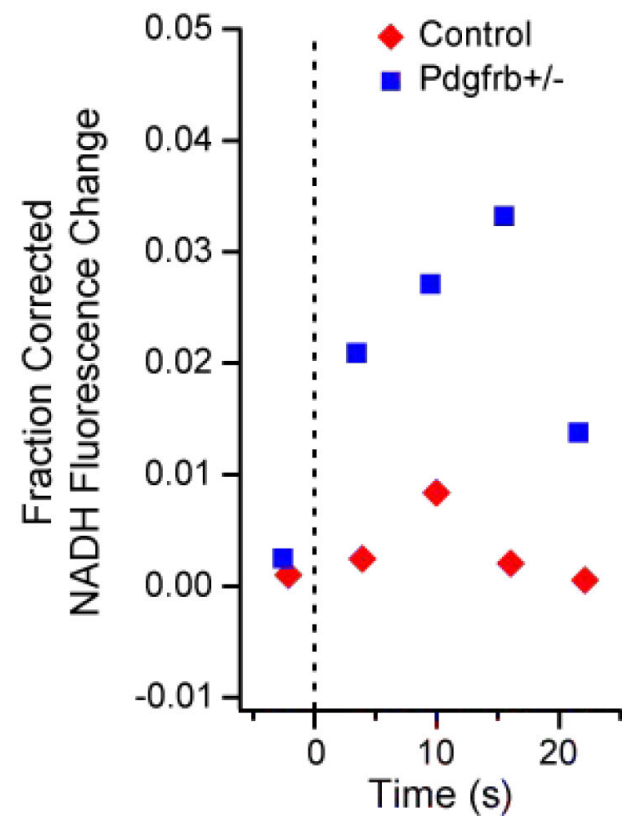

Figure 7: Two-photon measurements of NADH fluorescence intensity changes in response to stimulus in the somatosensory cortex of an anesthetized pericyte-deficient mouse (carrying a single platelet-derived growth factor receptor $\beta$ allele; $P d g f r \beta^{+/-}$), which exhibits altered metabolic responses to stimulus.

(Step 18C) (a) Representative two-photon images of SR101 (red) and NADH (blue)

fluorescence were acquired in the somatosensory cortex, $50 \mu \mathrm{m}$ from the brain surface.

SR101 and NADH images were recorded over time before and in response to an electrical hind limb stimulus ( $10 \mathrm{~s}$ duration at $10 \mathrm{~Hz}, 2 \mathrm{~ms}$ pulses). Representative images before (baseline) and at the time of the NADH peak fluorescence change are shown. NADH is intrinsically fluorescent, where increased fluorescence intensity typically correlates with the decreased cortical tissue oxygenation. SR101, a dye that labels astrocytes and oligodendrocytes, is added to the brain tissue and used as a non-functional control marker to correct for hemodynamic changes during stimulus. Scale bar is $20 \mu \mathrm{m}$. (b) Time course of NADH fluorescence before, and after an electrical hind limb stimulus starting at $0 \mathrm{~s}$. NADH fluorescence plotted over time is corrected for blood flow changes using the SR101 images as previously described ${ }^{105}$. Dashed line indicates stimulus start. The example shows data from one animal. For comparison, the corrected NADH signal time course from a control littermate animal is plotted. The response in the control animal has a much smaller amplitude compared to $P d g f r \beta^{+/-}$mouse, likely because the control animal better tolerates metabolic demand of the relatively short stimulus. This procedure was approved by the Institutional Animal Care and Use Committee at the University of Southern California with National Institutes of Health guidelines. 
a

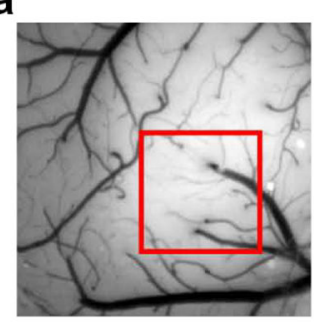

b

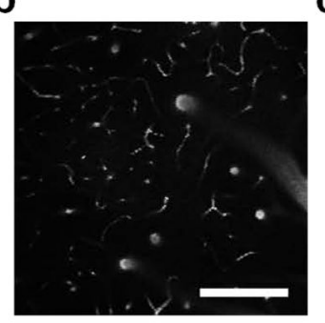

C

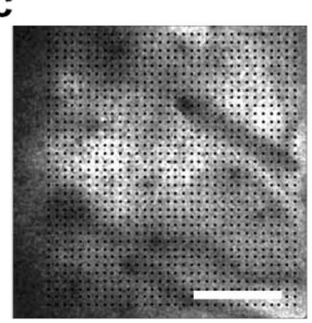

d

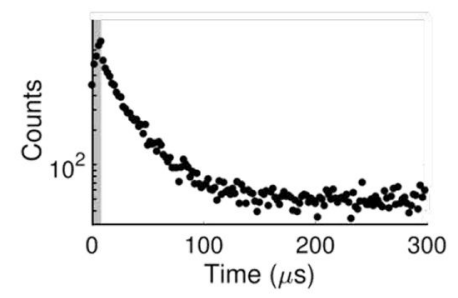

e
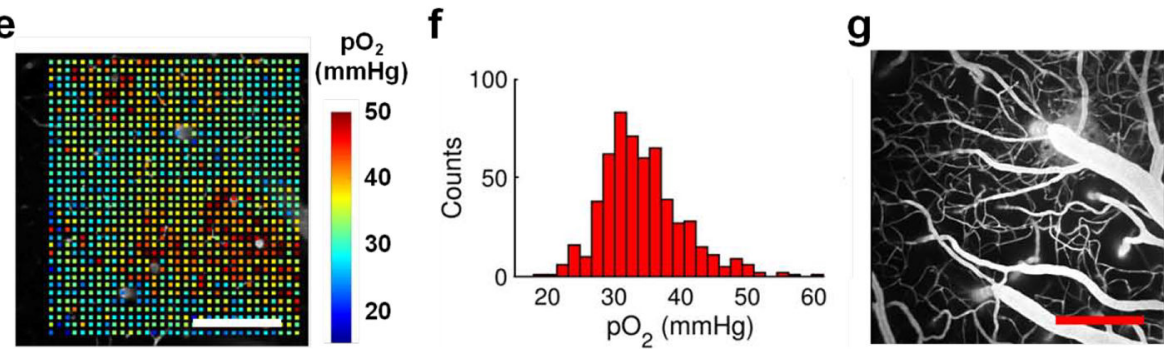

h

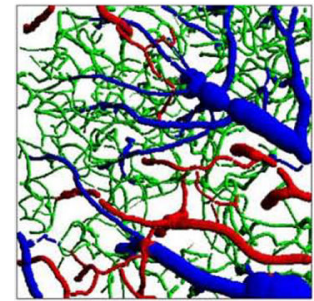

Figure 8: Two-photon acquisition of absolute tissue $\mathrm{pO}_{2}$ values in mouse cortex using PtP-C343 phosphorescent dye.

(Box 1) (a) Large field of view image of the pial vasculature inside the cranial window taken by a charge-couple device camera under green-light illumination is used to identify imaging locations. (b) A two-photon image of FITC-dextran (2000 kDa) labeled blood plasma 100 $\mu \mathrm{m}$ below the cortical surface at the location marked with the red square in a identifies the vascular structure at the region and depth selected for $\mathrm{pO}_{2}$ imaging. (c) Phosphorescence intensity survey image at the same location as $\mathbf{b}$ illustrating regional phosphorescence probe labeling. Black dots represent locations pre-selected for phosphorescence lifetime measurements. (d) Phosphorescence lifetime measurement from one of the grid point locations. 2000 decays were combined to generate the curve. Shaded area indicates $10 \mu \mathrm{s}$ excitation. (e) Measured $\mathrm{pO}_{2}$ values overlaid on the microvascular structural image presented in the panel b. $\mathrm{pO}_{2}$ values (in $\mathrm{mmHg}$ ) are color coded. (f) Histogram analysis of the $\mathrm{pO}_{2}$ values in (e). (g) Microvascular angiogram at the location marked by the red square in a. A $300 \mu \mathrm{m}$-thick microvascular image stack obtained at the end of the experiment by two-photon imaging of the blood plasma relabeled with Rhodamine B-dextran (70 kDa) robustly labels and identifies the vasculature present in the vicinity of the $\mathrm{pO}_{2}$ measurements, providing a vascular map of the region. (h) Computer-rendered projection of the segmented three-dimensional microvascular image stack from $\mathbf{g}$ with morphologically identified arterioles, capillaries, and venules falsely colored in red, green, and blue, respectively. Scale bars: $200 \mu \mathrm{m}$. This procedure was approved by the Massachusetts General Hospital Subcommittee on Research Animal Care with National Institutes of Health guidelines. 


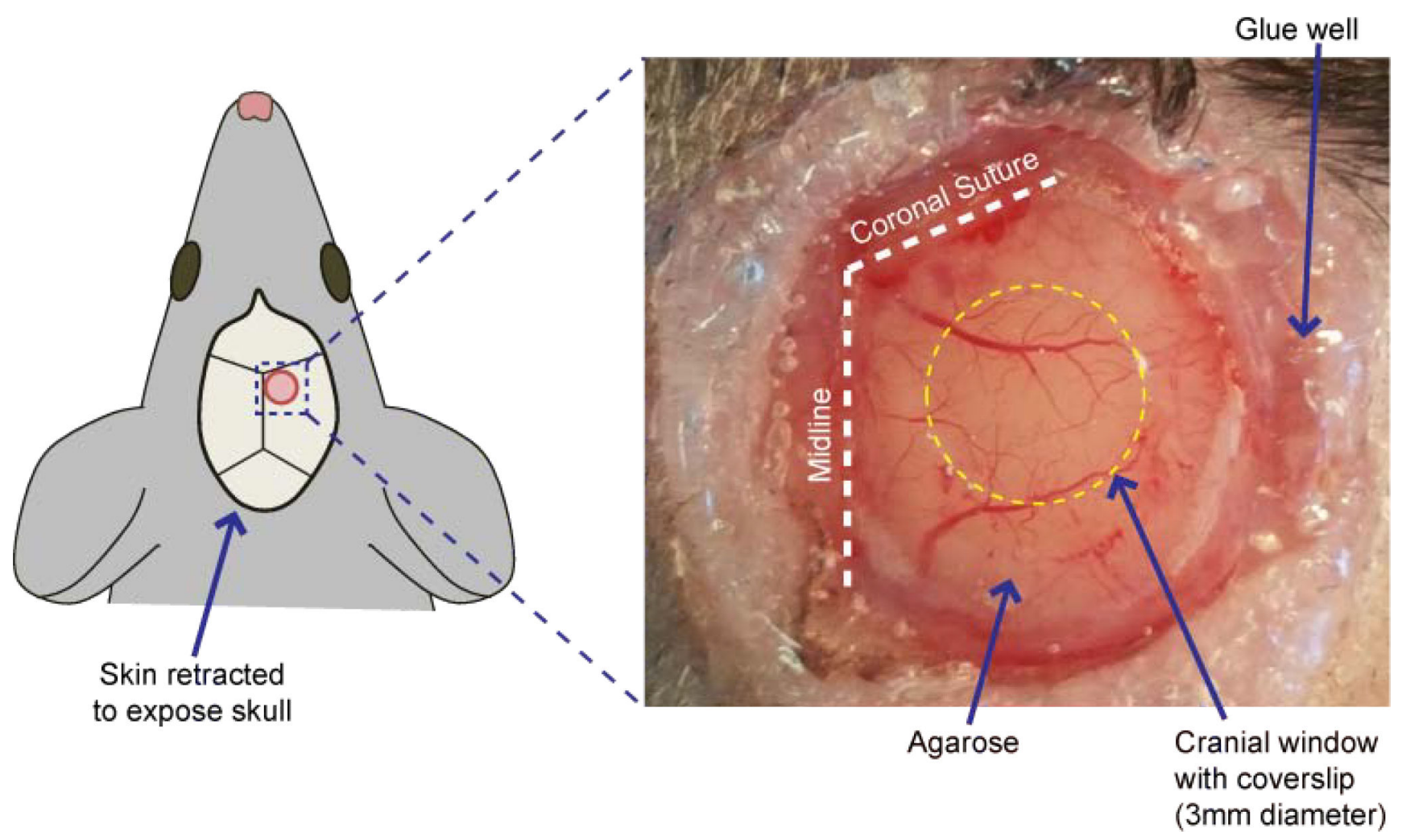

Figure 9: Cranial window preparation for in vivo imaging.

(Steps 1-17) In an anesthetized mouse, the skull over the hind limb somatosensory cortex is exposed (Left). A window is cut through the skull and carefully removed to expose the brain for imaging (red circle on left corresponds to the image on right). A glass coverslip window is then sealed to the brain using low melting temperature agarose (Right). A well formed of cyanoacrilate glue helps keep the agarose in place as it cools and solidifies. Notice the window is clear of blood, and all large surface vessels are intact. Approximate locations of midline and coronal sutures (intersecting at bregma) are shown. This procedure was approved by the Institutional Animal Care and Use Committee at the University of Southern California and the Massachusetts General Hospital Subcommittee on Research Animal Care with National Institutes of Health guidelines. 
TROUBLESHOOTING

\begin{tabular}{|c|c|c|c|}
\hline Step & Problem & Possible reason & Possible solution \\
\hline $\begin{array}{l}\text { Box } 2 \\
\text { step 4A.iii }\end{array}$ & $\begin{array}{l}\text { Motor not vibrating } \\
\text { when triggered }\end{array}$ & Insufficient trigger voltage & $\begin{array}{l}\text { This motor requires a } 5 \mathrm{~V} \text { trigger amplitude. If the camera } \\
\text { output trigger has smaller amplitude, a device to step up the } \\
\text { voltage to } 5 \mathrm{~V} \text { is required between the camera stimulus output } \\
\text { and the stimulus motor cable. }\end{array}$ \\
\hline \multirow[t]{5}{*}{ 18A.viii } & Vibrations in image & $\begin{array}{l}\text { Incorrect anesthesia level, resulting } \\
\text { in mouse movement }\end{array}$ & $\begin{array}{l}\text { Check depth of anathesia. Mouse should not respond to toe } \\
\text { pinch. }\end{array}$ \\
\hline & & $\begin{array}{l}\text { Head not affixed securely in the } \\
\text { stereotaxic frame }\end{array}$ & Reposition animal in stereotaxic frame. \\
\hline & & $\begin{array}{l}\text { Window glass not well affixed to } \\
\text { skull }\end{array}$ & Agarose may have dried out. Replace agarose and window. \\
\hline & $\begin{array}{l}\text { Is the signal real and } \\
\text { due to stimulus? }\end{array}$ & $\begin{array}{l}\text { Sometimes the signal is weak, or } \\
\text { near the edge of the window or } \\
\text { image }\end{array}$ & $\begin{array}{l}\text { (1) Take a data set with the stimulus off. There should be no } \\
\text { change in signal on average. }\end{array}$ \\
\hline & & & $\begin{array}{l}\text { (2) Shift the position of the mouse laterally (left or right, } \\
\text { forwards or back) and acquire another data set with stimulus. } \\
\text { The signal position in the image should move with the mouse. }\end{array}$ \\
\hline 18C.xi & Image is dim & Weak fluorescent signal & $\begin{array}{l}\text { Create an average image from the whole image sequence, } \\
\text { adjust contrast if needed, and use the average image to draw } \\
\text { the ROI. Once saved to the ROI manager, it can be applied to } \\
\text { the original image sequence. }\end{array}$ \\
\hline \multirow[t]{3}{*}{$\begin{array}{l}\text { Box } 5 \\
\text { step } 15\end{array}$} & Weak signal & Low signal and/or high background & $\begin{array}{l}\text { Emission filter transmission band should cover a large fraction } \\
\text { of the PtP-C } 343 \text { emission spectra and should have very low } \\
\text { transmission (OD }=5 \text { or greater) in the other parts of visible and } \\
\text { near infrared spectra. }\end{array}$ \\
\hline & & Low EOM extinction ratio & $\begin{array}{l}\text { EOM extinction ratio should be greater than } 400 \text {. Realign } \\
\text { EOM following manufacturer instructions to get a better ratio. }\end{array}$ \\
\hline & & Background light in room & $\begin{array}{l}\text { Carefully enclose microscope to prevent exposure of the } \\
\text { detector to any source of light from the room. Darken light- } \\
\text { producing elements in room. }\end{array}$ \\
\hline
\end{tabular}

